\title{
EFFECT OF WATERMELON PROPAGATION BY CUTTINGS ON VEGETATIVE GROWTH, YIELD AND FRUIT QUALITY
}

\author{
EL-ESLAMBOLY, A. A. S. A. \\ Protected Cultivation Dept., Horticulture Research Institute, ARC, Giza, Egypt
}

(Manuscript received 8 April 2014)

\begin{abstract}
Watermelon (Citrullus lanatus) is an important vegetable crop grown in Egypt. Seedless watermelon hybrids are preferred by most consumers because of their sweet taste and lack of hard seeds. The seedless watermelon (triploid hybrid watermelon) seeds are very expensive, do not germinate well and show least homogenous germination and growth. For these reasons, the present study aims to propagate seedless watermelon by cuttings to minimize the amounts of imported watermelon hybrid seeds in general and seedless watermelon in particular and reducing the cost of production and producing homogenous plants. Cutting is a method of asexual plant propagation widely used in horticulture. It is most commonly used for the propagation of trees and shrubs grown commercially. In vegetable crops cuttings are used on sweet potato and cassava as a commercial propagation method and cuttings can be used as a commercial propagation method in tomato, potato, eggplant, cucumber, melon and watermelon. The seedless watermelon cv. Yellow Buttercup "QV 766" F1 (yellow flesh) and SSX 7402 F1 hybrid (red flesh)) and seeded watermelon Cv. Aswan F1 hybrid were grown in plastic house for use as a source of cuttings (mother plants). Two types of cuttings were use, the first type was cuttings from terminal growing point of the main stem and lateral branches $10-15 \mathrm{~cm}$ in length 0.45 and $0.6 \mathrm{~cm}$ in diameter and the second type was cuttings including one node, bud and leaf. The study was conducted in three different experiments using randomized complete blocks design with three replicates during the seasons of 2010/2011 and 2011/2012 at a private farm in El-Badrashein City. Each hybrid was conducted in a single experiment. Each experiment included two types of cuttings treated with three concentrations $(0,100$, $200 \mathrm{ppm}$ ) of IBA and planted in seedling trays or pots $\mathrm{No} .8 \mathrm{~cm}$. Twelve cutting treatments beside the control which was produced from true seeds were used in each experiment. The obtained results showed that shoot tip cuttings with any concentrations of IBA and planted in seedling trays or in pots treatments gave a lower survival percentage than cuttings including one node and treated with any concentration of IBA and planted in seedling trays or in pots. This may be due to the significant increment in number of roots in cutting treatments have one node compared with both cutting include shoot tip and control plants. All cutting treatments which included one node gave a higher survival percentage than the seeds germination in both seedless cV. Yellow Buttercup (QV 766) F1 and SSX 7402 F1 hybrid while, the highest seed germination were recorded in Aswan F1 in both seasons. All cutting treatments gave a significant increment in all vegetative characteristics after 45 days from transplanting and early yield compared with control. The number of days from transplanting to flowering showed significant decrease than the control. The plants from cuttings reached harvesting 15 days earlier than the control. No significant differences were recorded between all cutting treatments and the control plants in all vegetative growth, yield
\end{abstract}


components and fruit characteristics. All cutting treatments gave a significant increase in early yield when compared with control plants.

Kay words: Seedless watermelon, triploid, cuttings, propagation.

Corresponding Author: Ahmed Abd El-Hady Sayed Abd El- Wahab ElEslamboly, Protected Cultivation Dept., Horticulture Research Institute, ARC, Egypt E-mail: Azaz2005asd@yahoo.com

\section{INTRODUCTION}

Watermelon (Citrullus lanatus) is an important crop cultivated in Egypt. It is considered one of the cash crops beside beings a popular summer fruit. Egypt occupied the fourth ranking in watermelon production after China, Turkey and USSR respectively, (Allred and Lucier, 1990). The watermelon cultivated area was 164, 529 feddans with average yield of 12.3 (ton/ fed.) according to the statistical data of ministry of agriculture 2006.

Triploid hybrids were first reported in Japan in 1947 (Kihara, 1951). In Egypt, tetraploid and triploid watermelon were first produced by Abd El- Hafez (1963) and who used the gene markers in the development the production of seedless watermelon in 1969. In the United States, triploid production became significant only in the 1980s. Triploid watermelons fruit are smaller, have a tougher rind and a much longer shelf life and have considerably more resistance to fruit blotch than diploid watermelon fruits (Garret et al., 1995). Consumers in the USA have accepted the socalled seedless watermelon despite the higher price to gain high return.

Seedless watermelons are sterile hybrids which develop fruits, but with no seeds. Seeds are produced by crossing a normal watermelon diploid $(2 X=22$ chromosomes) as a male with one that has been changed genetically by the treatment with colchicine (tetraploid ( $4 \mathrm{X}=44$ chromosomes) as a female parent. The seeds from this cross produce plants that, when pollinated with pollen from normal plants, produce seedless watermelons fruits (Kihara, 1951).

Triploid seeds production areas are primarily located in developing countries because of low labor costs. Andrus (1971) proposed low-cost production of triploid seeds by tetraploid plants interspersed in rows of diploid pollinators. Cross-pollination will predominate because diploid pollen is more abundant and competitive. Mixtures of triploids and a few tetraploid seed are sold, tetraploid watermelons are later separated by hand at harvest, based on fruit shape and color. This also requires trained workers.

In 1995 in the United States, commercial growers were paying \$140-\$180per 1000 seed, several fold the price of diploid hybrid seed. These prices are offset in the market, where triploid fruit can bring significantly higher prices than diploid fruit (Adelberg et al., 1997). 
Despite costly inputs, triploid seeds do not germinate well. Having small, triploid embryos encased in a thick tetraploid seed coat. During germination, numerous air spaces can fill with water, inhibiting the respiration of the expanding embryo. The seed coat sticks tightly to the cotyledons and frequently keeps the emerging cotyledons from expanding and photosynthesizing. To optimize emergence, triploid seed should be scarified, planted flat and germinated at temperatures above $27{ }^{\circ} \mathrm{C}$ with careful moisture control and then drenched with warm water just after emergence to remove the still pliable seed coat. Four weeks after planting, the seedlings are ready for transplant to the field. Triploid seed can be germinated under field conditions if carefully pregerminated (Adelberg et al., 1997).

Use of triploid hybrids has provided a method for production of seedless fruit. Kihara began working on seedless watermelons in 1939, and had commercial triploid hybrids available 12 years later. The development of triploid cultivars add several problems to the process of watermelon breeding: extra time for the development of tetraploids, additional selection against sterility and fruit abnormalities, choice of parents for reduced seed coat production, the reduction in seed yield per acre obtained by seed companies, reduced seed vigor for the grower, and the necessity for diploid pollenizer taking one-third of the grower's production field (Adelberg et al., 1997).

In Egypt the seedless watermelon (triploid hybrid watermelon) seeds are very expensive. Its price is about $1.75-2.00$ L.E /seed. Moreover the maximum percent of germination is $70 \%$ (according to Syngenta Company). So the cultivated area of seedless watermelon is about 100 feddans from 164.529 feddans watermelon production according to statistical data of Ministry of Agriculture (2006). In this connection, the fruit price of seedless fruit is about five times the price of seeded watermelon fruits. So, an efficient method for developing tetraploids is needed.

Mason (2004) recorded that chemical hormones may be applied to stimulate the formation either of roots or foliage/shoot growth. Heating may be used to warm the root zone (bottom heat) and encourage faster growth of roots, periodic misting of the foliage will cool the top of the plant or prevent dehydration of the foliage. Auxins are involved in a variety of things including stem growth, root formation, inhibiting lateral bud development, fruit and leaf abscission, fruit development and activating cambium cells. Of all the hormones, auxins have the greatest effect on root formation in cuttings. Root formation and development in a cutting. Indole butyric acid (IBA) is artificial, the most widely used rooting hormone, used on its own or in combination with NAA. Auxins can be applied to plants as a powder, gel or liquid. Cuttings are frequently treated with hormones which encourage root development. 
Treating different plant cuttings by dipping in IBA at 500 to1000 ppm for few seconds promote adventitious roots formation, root number and root length (Robbins et al., 1983). The application of IBA alone increased root fresh weight especially with the lowest concentration of IBA. Mepiquat Chloride (MC) also increased the root fresh weight in the same auxin level the response may be due to the reduction of ethylene production (El-Abd, 1997).

El-Abd (1997) found that all IBA treatments were effective in increasing the number, length and fresh weight of adventitious roots in cucumber cuttings as compared with control cuttings. IBA increased ethylene production comparing with MC treatments which reduce ethylene production but enhanced root elongation.

The aim of the study was to develop new pattern of cuttings technique for watermelon plants propagation to minimize the amounts of imported watermelon seeds in general and seedless watermelon in particular. Also to study the effect of using cuttings for watermelon propagation on plants homogeneity, growth rate, early yield productivity, and the time from planting to harvesting in three watermelon hybrids(two seedless and one seeded).

\section{MATERIALS AND METHODS}

Plant cutting, also known as striking or cloning, is a technique for vegetative (asexually) propagating plants in which a piece of the stem or root of the source plant is placed in a suitable medium. The cuttings produce new roots, stems, or both, and thus becomes a new plant independent from the parent. Two types of cuttings were taken from the watermelon mother plants, which were cultivated in the plastic house as a mother plants, i.e., cuttings from terminal growing point of main stem and lateral branches $10-15 \mathrm{~cm}$ in length and $0.45-0.6 \mathrm{~cm}$ in diameter and cuttings included one node, bud and a leaf.

This study was conducted during 2010/2011 and 2011/2012 on three watermelon hybrids, two seedless and one seeded watermelon hybrids. Such hybrid was treated as a separated experiment. Each experiment included 13 treatments, i.e., two types of cuttings, three concentrations (0, 100, 200 ppm) IBA (3- Indole butyric acid) for cuttings treating before planting and culture containers, viz, speedling trays or in pots no. 8 


\section{Plant material}

\section{a. Watermelon hybrids}

Two seedless watermelon (C. lanatus) hydrids, viz. Yellow Buttercup (QV 766) $F_{1}$ (yellow flesh) and SSX $7402 F_{1}$ hybrid (red flesh)) and seeded watermelon cv. Aswan $F_{1}$ hybrid from Sakata Seed Company, Japan were used in the present study.

\section{b. Cuttings preparation}

Seedless and seeded watermelon seeds were sown in a greenhouse in Badrashein city on $7^{\text {th }}$ of October 2010 in the first season and $12^{\text {th }}$ of October 2011 in the second season in the foam speedling trays with 84 cells filled with a mixture of peat-moss, vermiculite and berlite at the ratio of $1: 1: 1(\mathrm{v} / \mathrm{v})$. Three hundred grams of ammonium sulphate, $400 \mathrm{~g}$ calcium superphosphate, $150 \mathrm{~g}$ potassium sulphate, $50 \mathrm{ml}$. nutrient solution and $50 \mathrm{gm}$ of a fungicide were added for each $50 \mathrm{~kg}$ of the peatmoss. Seedless and seeded watermelon seedlings transplanted on $12^{\text {th }}$ of November, 2010 and $18^{\text {th }}$ of November 2011 in a plastic house in Badrashein city as mother plants to obtain cuttings in both seasons. Mother plants were treated with the conventional agricultural practices i.e., irrigation, fertilization, pests and diseases management as recommended by the Ministry of Agriculture in Egypt. For producing good watermelon vegetative growth, all female flowers and fruits were removed. Healthy mother plants, free of pests and diseases especially virus diseases, were selected for cuttings collection at 75 days after transplanting. Suitable branches chosen for cuttings from the shoot tip, upper, middle and bottom nodal segments of all primary or secondary branches.

Each type of cutting i.e., shoot tip and one node cuttings were divided into three groups, the first group was treated with $200 \mathrm{ppm}$ of IBA, the second group was treated with $100 \mathrm{ppm}$. by dipping it in IBA solution followed by immersing the bases of the cuttings in MC (Mepiquat Chloride) solution at the concentration $100 \mathrm{ppm}$ and the third group was not treated with IBA but immersed in distilled water for 10 seconds. The treated and untreated cuttings were planted in speedling trays 84 cells and in pots no. 8 filled with previous culture mixture. Speedling trays and pots after planted were removed immediately into the shaded plastic low tunnel for healing and root formation from the node region that was key factors for the survival of cutting plants. A polyethylene sheet was laid on the floor of the low tunnels and covered with a shallow layer of water. Speedling trays were placed on bricks to support the plants above the water layer. The plastic tunnel was closed to achieve a temperature of 25$32{ }^{\circ} \mathrm{C}$ and $(>85 \% \mathrm{RH})$ humidity for five to seven days. Watermelon plants which formed from cuttings were moved out of the tunnel and placed into a screenhouse, for seven days. 
The control plant was cultured by seeds from all cultivars were sown in speedling trays in the nursery at $2^{\text {nd }}$ of January 2011 in the first season and $24^{\text {th }}$ of December 2011 in the second season.

Watermelon seedlings were transplanted on $11^{\text {th }}$ February, 2011 in the open field in a private farm in El-Badrashein City, Giza in the first season and on the $8^{\text {th }}$ of February 2012 in the second season. 2200 Seedlings were transplanted in the feddan, $70 \%$ seedless and 30\% seeded (pollinator plants), in rows 5 meter length and 2 meter width. The space between plants was $1 \mathrm{~m}$. The single treatment contained 10 plants in two rows for seedless and one row from Aswan plants as pollinator. The conventional agricultural practices i.e., irrigation, fertilization, and weeding and pest control followed standard commercial practices, were done as recommended by the Ministry of Agriculture in Egypt, for watermelon production. Plots were first harvested on the $25^{\text {th }}$ of April in 2011 and 2012. The other harvesting, were done where the fruits were ripen. Three harvest times were conducted in the first and second seasons.

\section{Experimental design and statistical analysis}

Three experiments were conducted in randomized complete blocks design with three replications. Data were statistically analyzed using analyses of variance by the technique of analysis of variance ANOVA, with the Stat soft statistical MSTATC software program (Michigan State University, East Lansing, MI, USA). Probabilities of significance among treatments and means were compared with least significant difference L.S.D. $(P \leq 0.05)$ were used to compare means within and among treatments according to Gomez and Gomez (1984).

\section{Studied characteristics}

a. Survival rates: Survival rate was measured after 10-12 days from the cuttings by counting the surviving seedlings and dividing on the total number.

b. Number of root formed from the cuttings after 12 days from planting.

The following data were recorded during growth period until the end of harvesting.

\section{c. Vegetative growth characteristics}

Vegetative growth characters were recorded after 45 days from transplanting and at the end of harvesting in samples of four plants randomly chosen from each plot as follows
1. Plant length $(\mathrm{cm})$
2. Leaves area $\left(\mathrm{cm}^{2}\right)$ : 
It was expressed as the mean leaf area in $\mathrm{cm}^{2}$ using the dry weight method.

The leaves were cleaned from dust and then weighed to nearest $0.001 \mathrm{~g}$.

Therefore 20 disks of known area were separated, dried and weighed.

leaves area $\left(\mathrm{cm}^{2}\right)=\frac{\text { Dry weight of plant leaves }}{\text { Dry weight of } 20 \text { disks }} \times 20 \times$ the area of disk

Where, the area of a disk is about $1.0 \mathrm{~cm}$

3. Number of leaves per plant

4. Number of branches/plant

5. Plant fresh weight $(\mathrm{kg})$

6. Plant dry weight $(\mathrm{g})$

\section{d. Flowering}

1. Number of days to appearance of the first flower: Determined by calculating the days from transplanting in open field to the appearance of the first flower.

e. Yield and its components: The fruits were harvested 75 days after transplanting as a first harvest and the other harvesting were implemented where the fruits were ripen.

The following traits were evaluated

1. Early yield (ton/ feddan)

2. Total yield (ton/ feddan)

3. Total weight of fruits /plant

4. Marketable yield: It was determined by removing the abnormal fruits, fruit cracked, smitten fruits with any diseases and misshapen fruits which were considered culls as well as the fruit less than $3.0 \mathrm{~kg}$ from the total yield.

f. Fruit characteristics: Fruit characteristics were determined by measuring the following measurements

1. Average fruit weight $(\mathrm{kg})$

2. Fruit rind thickness $(\mathrm{mm})$ : Using a caliper.

3. Fruit size $\left(\mathrm{cm}^{3}\right)$ : It was measured by using water displacement technique by displacement the same size from water in normative beaker and estimated the same size from water. This was done in a special container which was filled with water until overflows form the spout. Fresh fruits were immersed and the overflow water volume was measured in a graduated cylinder. 


\section{g. Chemical determinations}

1. Panel test: Panel test was determined by panel testing (flavor) by 3 persons per replication with 3 replication based on the criterion, where $5=$ Excellent, $4=$ Good, 3= Medium, 2=Fair and 1= Poor

2. Total soluble solids (T.S.S. \%): It was measured in fruit juice by using a hand refractometer. This was estimated according to the methods described in A.O.A.C. (1975).

3. Dry matter percentage of fruit flesh: It was determined by allowing $100 \mathrm{~g}$ of fruit flesh to dry in an oven at $70^{\circ} \mathrm{C}$ till a constant weight.

4. Total sugars content: was determined in each fruit sample according to the method of Malik and Singh (1980).

5. Determination of leaf pigments: Green color reading: It was determined by using a SPAD 501 leaf chlorophyll meter (for using the greenness measurements) for fully expanded leaves (the fifth leaf from the shoots growing tip without destroying them) (Yadva, 1986).

\section{RESULTS AND DISCUSSION}

\section{Survival percentage}

This study provides the success of producing plants from two cutting types i.e., shoot tip and cuttings including one node treated with three concentrations of IBA ( 0 , $100,200 \mathrm{ppm}$ ). These cuttings were cultured in speedling trays having 84 cells or pots $8 \mathrm{~cm}$. The survival percentage of plants produced from plant cuttings (shoot tip and one node cuttings) from mother plants of all seedless and seeded cultivars and treated with three concentrations of IBA followed by planted in speedling trays and pots compared with germination ratio of all seedless and seeded cultivar are presented in Table (1).

Data in Table 1 showed significant differences between the two types of cuttings in survival percentage. The lowest survival percentage (58.24 and 61.15\%) were recorded in Aswan hybrid when using shoot tip cutting treated with zero concentration from IBA in both seasons. The same result was obtained for the hybrid SSX 7402 in the second season. On the other hand, the survival percentage of cuttings which include one node treated with any concentrations of IBA followed by planting in speedling trays or pots showed the highest value which ranged between $94.74 \%$ and $98.46 \%$ in all cultivars in both seasons. The highest value of seed germination (99\%) was obtained from Aswan watermelon whereas it ranged from 75 to $83 \%$ in seedless cultivars Buttercup and SSX7402. 
No significant differences were detected between both types of containers in all characteristics in both seasons.

The lowest survival percentage was observed in shoot tip cuttings treated with distilled water without IBA in both containers, which were $61.31 \%$ in both cultivars (SSX7402 and buttercup) in both seasons. As for number of roots data showed insignificant differences among shoot tip cuttings treated with different concentration of IBA in both containers in the first and second season. Data in this Table also showed significant increment in number of roots for cuttings with one node compared with shoot tip cutting. The highest value of the roots number formed from cuttings were recorded when cuttings included one node while the lowest value was observed when using cutting of shoot tip. These results were true regardless of the concentrations of IBA or containers. These results were true in all cultivars in both seasons. The survival percentage of plant cuttings depends on the success root formation which depends on the nutrients, hormones, quality and physiological case in these cuttings of the mother plants and the management conditions in the period after planting (Hartmann et al., 2002). These results were in agreement with that recorded by El-Abd, (1997) and Mason (2004).

Table 1. Effect of cutting types treated with three concentrations of IBA and planted in two containers compared with true seeds germination of three watermelon hybrids on survival percentage and number of roots in 2011 and 2012 seasons.

\begin{tabular}{|c|c|c|c|c|c|c|c|c|c|c|c|c|c|c|}
\hline \multirow{2}{*}{\multicolumn{3}{|c|}{ Characteristics }} & \multicolumn{6}{|c|}{ Survival percentage \% } & \multicolumn{6}{|c|}{ Number of roots } \\
\hline & & & \multicolumn{6}{|c|}{ Cultivars } & \multicolumn{6}{|c|}{ Cultivars } \\
\hline \multicolumn{3}{|c|}{ Treatments } & \multicolumn{2}{|c|}{ Buttercup } & \multicolumn{2}{|c|}{ SSX 7402} & \multicolumn{2}{|c|}{ Aswan } & \multicolumn{2}{|c|}{ Buttercup } & \multicolumn{2}{|c|}{ SSX 7402} & \multicolumn{2}{|c|}{ Aswan } \\
\hline & & $\begin{array}{l}\text { IBA } \\
\text { ppm }\end{array}$ & 2011 & 2012 & 2011 & 2012 & 2011 & 2012 & 2011 & 2012 & 2011 & 2012 & 2011 & 2012 \\
\hline \multicolumn{3}{|c|}{ Control (seeds) } & 83.2 & 79.4 & 75.4 & 81.1 & 99.0 & 97.1 & 1 & 1 & 1 & 1 & 1 & 1 \\
\hline \multirow{6}{*}{ 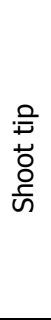 } & \multirow{3}{*}{ Trays } & 0 & 61.3 & 61.9 & 62.7 & 61.3 & 62.1 & 66.3 & 5.21 & 5.39 & 5.67 & 5.29 & 4.35 & 4.05 \\
\hline & & 100 & 64.5 & 68.1 & 66.6 & 65.5 & 72.3 & 73.2 & 6.19 & 6.15 & 6.72 & 6.06 & 4.75 & 5.20 \\
\hline & & 200 & 68.1 & 69.8 & 69.3 & 67.3 & 74.2 & 61.2 & 7.19 & 6.34 & 6.90 & 6.82 & 5.35 & 5.05 \\
\hline & \multirow{3}{*}{$\begin{array}{l}\text { Pots } \\
8 \mathrm{~cm}\end{array}$} & 0 & 62.3 & 62.2 & 62.7 & 61.9 & 58.2 & 69.2 & 5.31 & 5.53 & 5.68 & 5.33 & 5.75 & 6.05 \\
\hline & & 100 & 66.9 & 67.6 & 68.1 & 66.2 & 79.3 & 73.1 & 6.36 & 6.15 & 6.50 & 6.29 & 5.05 & 5.75 \\
\hline & & 200 & 69.5 & 69.9 & 70.2 & 68.8 & 77.2 & 72. 2 & 7.31 & 6.68 & 6.61 & 6.44 & 6.15 & 6.35 \\
\hline \multirow{6}{*}{ 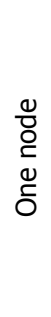 } & \multirow{3}{*}{ Trays } & 0 & 95.1 & 94.8 & 94.7 & 95.1 & 98.3 & 96.2 & 9.17 & 8.97 & 8.25 & 8.54 & 7.75 & 8.05 \\
\hline & & 100 & 96.5 & 96.4 & 96.7 & 96.5 & 97.3 & 97.1 & 9.66 & 9.42 & 8.97 & 9.22 & 8.05 & 8.05 \\
\hline & & 200 & 97.5 & 97.7 & 96.8 & 97.5 & 96.2 & 98.2 & 9.86 & 9.60 & 9.55 & 9.77 & 8.75 & 9.05 \\
\hline & \multirow{3}{*}{$\begin{array}{l}\text { Pots } \\
8 \mathrm{~cm}\end{array}$} & 0 & 95.5 & 95.7 & 95.1 & 93.9 & 97.2 & 98.3 & 8.92 & 9.06 & 8.25 & 8.81 & 8.05 & 8.35 \\
\hline & & 100 & 97.2 & 97.6 & 96.5 & 95.9 & 98.3 & 97.2 & 9.12 & 9.59 & 9.35 & 9.13 & 9.35 & 9. 50 \\
\hline & & 200 & 98.4 & 98.4 & 98.0 & 98.5 & 98.3 & 97.2 & 10.08 & 10.14 & 9.75 & 9.94 & 9.75 & 10.25 \\
\hline \multicolumn{3}{|c|}{ L.S.D. 0.05} & 14.2 & 11.2 & 12.8 & 13.4 & 10.9 & 11.4 & 3.36 & 3.00 & 2.73 & 2.81 & 2.74 & 2.62 \\
\hline
\end{tabular}




\section{Vegetative growth characteristics}

Data in Tables 2,3and 4 indicated that there was significant increment in plant vegetative growth which was obtained from cuttings compared with watermelon plants produced from seeds (control). These results were true in the three, i.e. seedless cultivars Buttercup F1and SSX7402 F1 and seeded hybrid "AswanF1" in both seasons. This appeared in plant length, leaf area and number of branches and leaves in the first 45 days after planting. These results may be due to the increment in the root formation from cuttings when compared with the primary root which formed from seeds. Cuttings formed large number of lateral roots from the root initiation at the nodes in cuttings. On the other hand data in Tables 5, 6 and 7 showed that at the harvesting stage, there were no significant differences between vegetative growth characteristics, i.e. plant length, leaves area, number of branches and leaves and plant fresh and dry weight of watermelon plants from different treated cuttings and that obtained from seeds (control). These results were evidence in all cultivars under this study during the two seasons.

Table 2. Effect of using cuttings treated with three concentrations of IBA in two containers compared with true seeds on some vegetative growth characteristics of Aswan hybrid after 45 days from planting in 2011 and 2012 seasons.

\begin{tabular}{|c|c|c|c|c|c|c|c|c|c|c|}
\hline \multicolumn{3}{|c|}{ Characteristics } & \multicolumn{4}{|c|}{2011} & \multicolumn{4}{|c|}{2012} \\
\hline \multirow{2}{*}{\multicolumn{2}{|c|}{ Treatments }} & \multirow{2}{*}{$\begin{array}{l}\text { IBA } \\
\text { ppm }\end{array}$} & \multirow{3}{*}{ 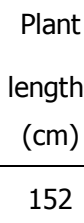 } & \multirow{3}{*}{\begin{tabular}{|c|}
$\begin{array}{c}\text { Leaf area } \\
\left(\mathrm{cm}^{2}\right)\end{array}$ \\
132.65
\end{tabular}} & \multicolumn{2}{|c|}{ Number/plant } & \multirow{2}{*}{$\begin{array}{l}\text { Plant } \\
\text { length } \\
(\mathrm{cm})\end{array}$} & \multirow{2}{*}{$\begin{array}{c}\text { Leaf area } \\
\left(\mathrm{cm}^{2}\right)\end{array}$} & \multicolumn{2}{|c|}{ Number /plant } \\
\hline & & & & & Branchec & Iอวหา & & & Branches & I Рวмс \\
\hline & \multicolumn{2}{|c|}{ Control from seeds } & & & 5.5 & 111.8 & 137 & 136.43 & 6.0 & 107.6 \\
\hline \multirow{6}{*}{ 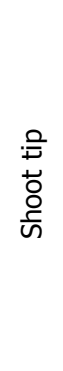 } & \multirow{3}{*}{$\begin{array}{r}\text { Speedlin } \\
\text { Trays }\end{array}$} & 0 & 186 & 165.68 & 7.3 & 128.8 & 168 & 189.22 & 7.5 & 135.5 \\
\hline & & 100 & 189 & 181.13 & 7.0 & 136.4 & 182 & 188.93 & 7.5 & 143.7 \\
\hline & & 200 & 183 & 188.57 & 7.0 & 141.1 & 188 & 178.9 & 6.3 & 141.4 \\
\hline & \multirow{3}{*}{$\mathrm{Pc}$} & 0 & 191 & 175.20 & 7.3 & 125.2 & 189 & 192.28 & 8.0 & 145.1 \\
\hline & & 100 & 209 & 187.16 & 7.0 & 147.7 & 210 & 190.89 & 7.0 & 139.6 \\
\hline & & 200 & 193 & 181.47 & 6.7 & 151.0 & 216 & 194.23 & 7.0 & 129.8 \\
\hline \multirow{6}{*}{ 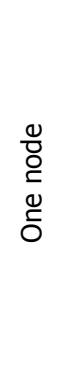 } & \multirow{3}{*}{$\begin{array}{r}\text { Speedlin } \\
\text { Trays }\end{array}$} & 0 & 231 & 216.09 & 8.0 & 154.3 & 223 & 232.66 & 8.5 & 144.3 \\
\hline & & 100 & 197 & 225.68 & 8.0 & 147.7 & 203 & 239.41 & 8.0 & 148.7 \\
\hline & & 200 & 235 & 229.94 & 7.0 & 150.5 & 237 & 225.65 & 8.0 & 159.9 \\
\hline & & 0 & 222 & 229.99 & 8.3 & 145.9 & 217 & 218.48 & 8.0 & 152.4 \\
\hline & & 100 & 231 & 235.85 & 8.0 & 160.3 & 235 & 233.14 & 8.5 & 157.2 \\
\hline & & 200 & 236 & 227.40 & 7.0 & 157.9 & 227 & 233.63 & 8.0 & 154.9 \\
\hline \multicolumn{3}{|c|}{ L.S.D. 0.05} & 24.5 & 31.240 & 1.7 & 16.10 & 30.1 & 36.210 & 1.2 & 23.45 \\
\hline
\end{tabular}


Table 3. Effect of using cuttings treated with three concentrations of IBA in two containers compared with true seeds on some vegetative growth characteristics of Buttercup hybrid after 45 days from planting in 2011 and 2012 seasons.

\begin{tabular}{|c|c|c|c|c|c|c|c|c|c|c|}
\hline \multicolumn{3}{|c|}{ Characteristics } & \multicolumn{4}{|c|}{2011} & \multicolumn{4}{|c|}{2012} \\
\hline \multirow{2}{*}{\multicolumn{2}{|c|}{$\begin{array}{c}\text { Treatmen } \\
\text { ts }\end{array}$}} & \multirow{2}{*}{$\begin{array}{l}\text { IBA } \\
\mathrm{ppm}\end{array}$} & \multirow{2}{*}{$\begin{array}{l}\text { Plant } \\
\text { length } \\
(\mathrm{cm})\end{array}$} & \multirow{2}{*}{$\begin{array}{l}\text { Leaf } \\
\text { area } \\
\left(\mathrm{cm}^{2}\right)\end{array}$} & \multicolumn{2}{|c|}{ Number/plant } & \multirow{2}{*}{$\begin{array}{l}\text { Plant } \\
\text { length } \\
(\mathrm{cm})\end{array}$} & \multirow{2}{*}{$\begin{array}{l}\text { Leaf } \\
\text { area } \\
\left(\mathrm{cm}^{2}\right)\end{array}$} & \multicolumn{2}{|c|}{ Number /plant } \\
\hline & & & & & Branches & Leaves & & & Branches & Leaves \\
\hline & Control frol & seeds & 143 & 149.47 & 5.3 & 100.9 & 151 & 141.58 & 6.0 & 112.8 \\
\hline \multirow{6}{*}{$\begin{array}{l}\stackrel{0}{ \pm} \\
\stackrel{0}{0} \\
\frac{0}{n}\end{array}$} & \multirow{3}{*}{$\begin{array}{c}\text { Speedling } \\
\text { Trays }\end{array}$} & 0 & 182 & 175.38 & 6.7 & 123.6 & 169 & 186.58 & 7.7 & 135.8 \\
\hline & & 100 & 179 & 183.57 & 7.0 & 129.6 & 189 & 179.57 & 7.3 & 139.4 \\
\hline & & 200 & 178 & 180.75 & 7.3 & 139.8 & 182 & 181.87 & 6.7 & 134.9 \\
\hline & \multirow{3}{*}{$\begin{array}{l}\text { Pots } \\
8 \mathrm{~cm}\end{array}$} & 0 & 186 & 186.25 & 6.7 & 131.7 & 191 & 185.78 & 7.3 & 143.8 \\
\hline & & 100 & 189 & 179.98 & 7.3 & 139.5 & 212 & 191.88 & 7.3 & 141.8 \\
\hline & & 200 & 190 & 191.67 & 7.0 & 149.6 & 209 & 196.98 & 7.7 & 148.7 \\
\hline \multirow{6}{*}{$\begin{array}{l}\frac{0}{0} \\
\frac{1}{c} \\
\stackrel{0}{0}\end{array}$} & \multirow{3}{*}{$\begin{array}{c}\text { Speedling } \\
\text { Trays }\end{array}$} & 0 & 218 & 229.09 & 7.7 & 157.8 & 227 & 241.54 & 8.7 & 143.8 \\
\hline & & 100 & 228 & 238.68 & 8.3 & 158.6 & 214 & 231.58 & 8.7 & 152.5 \\
\hline & & 200 & 226 & 234.94 & 7.3 & 148.5 & 229 & 229.74 & 8.3 & 149.2 \\
\hline & \multirow{3}{*}{$\begin{array}{l}\text { Pots } \\
8 \mathrm{~cm}\end{array}$} & 0 & 228 & 232.99 & 7.7 & 151.7 & 226 & 238.58 & 8.7 & 155.4 \\
\hline & & 100 & 219 & 227.66 & 8.7 & 161.8 & 248 & 241.74 & 8.0 & 148.4 \\
\hline & & 200 & 231 & 238.65 & 8.3 & 152.8 & 238 & 239.57 & 9.0 & 151.8 \\
\hline \multicolumn{3}{|c|}{ L.S.D. 0.05} & 32.4 & 22.240 & 1.3 & 22.00 & 26.9 & 39.800 & 1.5 & 22.00 \\
\hline
\end{tabular}

Table 4. Effect of using cuttings treated with three concentrations of IBA in two containers compared with true seeds on some vegetative growth characteristics of SSX7402 hybrid after 45 days from planting in 2011 and 2012 seasons.

\begin{tabular}{|c|c|c|c|c|c|c|c|c|c|c|}
\hline \multicolumn{3}{|c|}{ Characteristics } & \multicolumn{4}{|c|}{2011} & \multicolumn{4}{|c|}{2012} \\
\hline \multirow{2}{*}{\multicolumn{2}{|c|}{ Treatments }} & \multirow{2}{*}{$\begin{array}{l}\text { IBA } \\
\text { ppm }\end{array}$} & \multirow{3}{*}{$\begin{array}{c}\text { Plant } \\
\text { length }(\mathrm{cm})\end{array}$} & \multirow{3}{*}{ 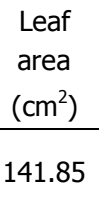 } & \multicolumn{2}{|c|}{ Number/plant } & \multirow{2}{*}{$\begin{array}{c}\text { Plant } \\
\text { length }(\mathrm{cm})\end{array}$} & \multirow{2}{*}{$\begin{array}{l}\text { Leaf } \\
\text { area } \\
\left(\mathrm{cm}^{2}\right)\end{array}$} & \multicolumn{2}{|c|}{ Number / plant } \\
\hline & & & & & Branches & Leaves & & & Branches & Leaves \\
\hline & \multicolumn{2}{|c|}{$\begin{array}{l}\text { Control from } \\
\text { seeds }\end{array}$} & & & 6.0 & 121.5 & 132 & 162.00 & 6.0 & 119.9 \\
\hline \multirow{6}{*}{$\begin{array}{l}\stackrel{0}{\rightleftharpoons} \\
\stackrel{+}{\circ} \\
\frac{o}{n}\end{array}$} & \multirow{3}{*}{$\begin{array}{c}\text { Speedling } \\
\text { Trays }\end{array}$} & 0 & 174 & 165.89 & 7.7 & 138.9 & 171 & 197.65 & 7.7 & 142.6 \\
\hline & & 100 & 182 & 178.36 & 7.3 & 139.9 & 177 & 201.57 & 7.0 & 140.6 \\
\hline & & 200 & 179 & 187.75 & 7.7 & 147.1 & 183 & 213.19 & 7.0 & 139.8 \\
\hline & \multirow{3}{*}{$\begin{array}{l}\text { Pots } \\
8 \mathrm{~cm}\end{array}$} & 0 & 189 & 173.86 & 7.3 & 137.6 & 190 & 218.56 & 8.3 & 146.4 \\
\hline & & 100 & 205 & 183.92 & 7.3 & 143.1 & 201 & 124.54 & 8.0 & 140.3 \\
\hline & & 200 & 211 & 199.69 & 7.3 & 152.0 & 209 & 214.45 & 8.0 & 143.8 \\
\hline \multirow{6}{*}{$\begin{array}{l}\frac{0}{0} \\
\frac{1}{c} \\
\stackrel{0}{0} \\
0\end{array}$} & \multirow{3}{*}{$\begin{array}{c}\text { Speedling } \\
\text { Trays }\end{array}$} & 0 & 238 & 209.95 & 8.7 & 158.1 & 228 & 234.54 & 8.3 & 147.6 \\
\hline & & 100 & 209 & 235.68 & 8.3 & 146.0 & 222 & 242.47 & 8.3 & 151.5 \\
\hline & & 200 & 222 & 229.87 & 8.0 & 151.6 & 231 & 240.15 & 8.7 & 149.7 \\
\hline & \multirow{3}{*}{$\begin{array}{l}\text { Pots } \\
8 \mathrm{~cm}\end{array}$} & 0 & 230 & 242.96 & 8.0 & 150.6 & 227 & 229.48 & 8.7 & 158.8 \\
\hline & & 100 & 228 & 237.87 & 8.7 & 160.1 & 236 & 236.05 & 8.3 & 149.7 \\
\hline & & 200 & 237 & 236.85 & 8.0 & 153.3 & 232 & 235.15 & 8.3 & 156.8 \\
\hline \multicolumn{3}{|c|}{ L.S.D. 0.05} & 33.8 & 22.580 & 1.5 & 14.10 & 36.0 & 32.909 & 1.1 & 20.50 \\
\hline
\end{tabular}


Table 5. Effect of using cuttings treated with three concentrations of IBA in two containers compared with true seeds on some vegetative growth characteristics of Aswan hybrid at harvesting in 2011 and 2012 seasons.

\begin{tabular}{|c|c|c|c|c|c|c|c|c|c|c|c|c|}
\hline \multicolumn{3}{|c|}{ Characteristics } & \multicolumn{5}{|c|}{2011} & \multicolumn{5}{|c|}{2012} \\
\hline \multirow{2}{*}{\multicolumn{2}{|c|}{ Treatments }} & \multirow{2}{*}{$\begin{array}{l}\text { IBA } \\
\text { ppm }\end{array}$} & \multirow{2}{*}{$\begin{array}{c}\text { Plant } \\
\text { length }(\mathrm{cm})\end{array}$} & \multirow{2}{*}{$\begin{array}{c}\text { Plant fresh } \\
\text { weight }\end{array}$} & \multirow{2}{*}{$\begin{array}{c}\text { Leaves area } \\
\left(\mathrm{cm}^{2}\right)\end{array}$} & \multicolumn{2}{|c|}{ Number/plant } & \multirow{2}{*}{$\begin{array}{c}\text { Plant } \\
\text { length }(\mathrm{cm})\end{array}$} & \multirow{2}{*}{$\begin{array}{c}\text { Plant fresh } \\
\text { weight }\end{array}$} & \multirow{2}{*}{$\begin{array}{c}\text { Leaves area } \\
\left(\mathrm{cm}^{2}\right)\end{array}$} & \multicolumn{2}{|c|}{ Number /plant } \\
\hline & & & & & & Branches & Leaves & & & & Branches & Leaves \\
\hline \multicolumn{3}{|c|}{ Control from seeds } & 508.0 & 2.493 & 57167 & 12.7 & 512.6 & 489.3 & 2.403 & 55257 & 12.5 & 513.3 \\
\hline \multirow{6}{*}{$\begin{array}{l}\stackrel{ }{ \pm} \\
+ \\
\frac{0}{0} \\
\frac{5}{n}\end{array}$} & \multirow{3}{*}{$\begin{array}{c}\text { Speedling } \\
\text { Trays }\end{array}$} & 0 & 499.3 & 2.467 & 56740 & 11.6 & 489.0 & 499.6 & 2.450 & 53935 & 11.8 & 485.0 \\
\hline & & 100 & 501.0 & 2.390 & 59062 & 11.6 & 491.3 & 491.6 & 2.380 & 57440 & 11.5 & 500.0 \\
\hline & & 200 & 495.0 & 2.460 & 56800 & 11.2 & 485.0 & 481.6 & 2.417 & 57898 & 11.8 & 481.3 \\
\hline & \multirow{3}{*}{$\begin{array}{l}\text { Pots } \\
8 \mathrm{~cm}\end{array}$} & 0 & 501.0 & 2.387 & 56901 & 11.7 & 499.6 & 525.6 & 2.373 & 58153 & 12.0 & 484.6 \\
\hline & & 100 & 505.6 & 2.520 & 53636 & 11.5 & 481.0 & 478.6 & 2.367 & 57955 & 11.5 & 503.3 \\
\hline & & 200 & 496.0 & 2.440 & 54692 & 11.6 & 484.0 & 489.0 & 2.427 & 58307 & 11.9 & 503.0 \\
\hline \multirow{6}{*}{$\begin{array}{l}\frac{1}{0} \\
\frac{0}{C} \\
\text { ठ } \\
\text { ठ }\end{array}$} & \multirow{3}{*}{$\begin{array}{c}\text { Speedling } \\
\text { Trays }\end{array}$} & 0 & 510.3 & 2.443 & 54403 & 11.7 & 485.6 & 490.0 & 2.430 & 57170 & 11.6 & 492.0 \\
\hline & & 100 & 505.3 & 2.407 & 57019 & 11.6 & 492.6 & 495.0 & 2.407 & 58051 & 10.8 & 491.3 \\
\hline & & 200 & 525.0 & 2.440 & 57118 & 11.7 & 493.0 & 505.6 & 2.467 & 57739 & 11.3 & 499.3 \\
\hline & \multirow{3}{*}{$\begin{array}{l}\text { Pots } \\
8 \mathrm{~cm}\end{array}$} & 0 & 496.3 & 2.507 & 57435 & 11.5 & 487.6 & 490.6 & 2.480 & 55393 & 11.0 & 494.0 \\
\hline & & 100 & 516.0 & 2.503 & 56868 & 11.9 & 487.0 & 491.0 & 2.470 & 57834 & 11.4 & 498.0 \\
\hline & & 200 & 493.0 & 2.510 & 58511 & 12.0 & 494.7 & 491.6 & 2.453 & 57701 & 10.7 & 490.3 \\
\hline \multicolumn{3}{|c|}{ L.S.D. 0.05} & NS & NS & NS & NS & NS & NS & NS & NS & NS & NS \\
\hline
\end{tabular}


Table 6. Effect of using cuttings treated with three concentrations of IBA in two containers compared with true seeds on some vegetative growth characteristics of Buttercup hybrid at harvesting in 2011 and 2012 seasons.

\begin{tabular}{|c|c|c|c|c|c|c|c|c|c|c|c|c|}
\hline \multirow{2}{*}{\multicolumn{3}{|c|}{ Characteristics }} & \multicolumn{5}{|c|}{2011} & \multicolumn{5}{|c|}{2012} \\
\hline & & & \multirow{2}{*}{$\begin{array}{c}\text { Plant } \\
\text { length (cm) }\end{array}$} & \multirow{2}{*}{$\begin{array}{c}\text { Plant fresh } \\
\text { weight }\end{array}$} & \multirow{2}{*}{$\begin{array}{c}\text { Leaves } \\
\text { area }\left(\mathrm{cm}^{2}\right)\end{array}$} & \multicolumn{2}{|c|}{ Number/plant } & \multirow{2}{*}{$\begin{array}{c}\text { Plant } \\
\text { length }(\mathrm{cm})\end{array}$} & \multirow{2}{*}{$\begin{array}{c}\text { Plant fresh } \\
\text { weight }\end{array}$} & \multirow{2}{*}{$\begin{array}{l}\text { Leaves area } \\
\qquad\left(\mathrm{cm}^{2}\right)\end{array}$} & \multicolumn{2}{|c|}{ Number /plant } \\
\hline & atments & $\begin{array}{l}\text { IBA } \\
\text { ppm }\end{array}$ & & & & Branches & Leaves & & & & Branches & Leaves \\
\hline \multicolumn{3}{|c|}{ Control from seeds } & 379.07 & 2.167 & 46766 & 11.23 & 456.00 & 380.40 & 2.159 & 48001 & 11.57 & 482.47 \\
\hline \multirow{6}{*}{ 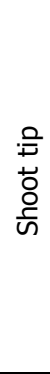 } & \multirow{3}{*}{$\begin{array}{c}\text { Speedling } \\
\text { Trays }\end{array}$} & 0 & 388.77 & 2.135 & 47620 & 10.77 & 444.13 & 391.50 & 2.167 & 47530 & 10.87 & 469.83 \\
\hline & & 100 & 375.23 & 2.120 & 49390 & 10.57 & 459.50 & 382.07 & 2.161 & 49337 & 11.00 & 478.07 \\
\hline & & 200 & 387.73 & 2.149 & 46552 & 10.70 & 436.23 & 379.63 & 2.199 & 50022 & 11.13 & 463.90 \\
\hline & \multirow{3}{*}{$\begin{array}{l}\text { Pots } \\
8 \mathrm{~cm}\end{array}$} & 0 & 374.03 & 2.122 & 47568 & 11.13 & 448.20 & 404.27 & 2.171 & 49771 & 11.47 & 466.17 \\
\hline & & 100 & 378.27 & 2.173 & 45663 & 10.67 & 447.07 & 377.97 & 2.142 & 49111 & 11.23 & 474.67 \\
\hline & & 200 & 383.00 & 2.129 & 45978 & 10.63 & 446.43 & 388.17 & 2.206 & 50017 & 10.93 & 471.07 \\
\hline \multirow{5}{*}{ 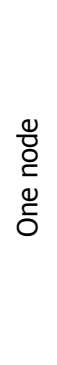 } & \multirow{2}{*}{$\begin{array}{c}\text { Speedling } \\
\text { Trays }\end{array}$} & 0 & 381.47 & 2.121 & 46495 & 10.97 & 453.40 & 388.60 & 2.187 & 49777 & 11.13 & 475.00 \\
\hline & & 200 & 385.90 & 2.157 & 47475 & 10.70 & 464.97 & 387.53 & 2.218 & 50281 & 10.83 & 480.03 \\
\hline & \multirow{3}{*}{$\begin{array}{l}\text { Pots } \\
8 \mathrm{~cm}\end{array}$} & 0 & 393.17 & 2.164 & 47587 & 10.87 & 448.27 & 382.37 & 2.201 & 48091 & 10.73 & 477.77 \\
\hline & & 100 & 380.83 & 2.198 & 46341 & 11.00 & 459.83 & 381.17 & 2.210 & 49872 & 11.03 & 477.73 \\
\hline & & 200 & 390.13 & 2.172 & 47818 & 10.90 & 455.70 & 385.87 & 2.213 & 49019 & 10.77 & 477.47 \\
\hline \multicolumn{3}{|c|}{ L.S.D. 0.05} & NS & NS & NS & NS & NS & NS & NS & NS & NS & NS \\
\hline
\end{tabular}


Table 7. Effect of using cuttings treated with three concentrations of IBA in two containers compared with true seeds on some vegetative growth characteristics of SSX 7402 hybrid at harvesting in 2011 and 2012 seasons.

\begin{tabular}{|c|c|c|c|c|c|c|c|c|c|c|c|c|}
\hline \multirow{2}{*}{\multicolumn{3}{|c|}{ Characteristics }} & \multicolumn{5}{|c|}{2011} & \multicolumn{5}{|c|}{2012} \\
\hline & & & \multirow{2}{*}{$\begin{array}{c}\text { Plant } \\
\text { length (cm) }\end{array}$} & \multirow{2}{*}{$\begin{array}{c}\text { Plant fresh } \\
\text { weight }\end{array}$} & \multirow{2}{*}{$\begin{array}{l}\text { Leaves area } \\
\qquad\left(\mathrm{cm}^{2}\right)\end{array}$} & \multicolumn{2}{|c|}{ Number/plant } & \multirow{2}{*}{$\begin{array}{c}\text { Plant } \\
\text { length }(\mathrm{cm})\end{array}$} & \multirow{2}{*}{$\begin{array}{c}\text { Plant fresh } \\
\text { weight }\end{array}$} & \multirow{2}{*}{$\begin{array}{c}\text { Leaves area } \\
\qquad\left(\mathrm{cm}^{2}\right)\end{array}$} & \multicolumn{2}{|c|}{ Number /plant } \\
\hline & atments & IBA & & & & Branches & Leaves & & & & Branches & Leaves \\
\hline \multicolumn{3}{|c|}{ Control from seeds } & 337.7 & 2.255 & 45323 & 12.0 & 486.7 & 344.3 & 2.328 & 49939 & 12.8 & 544.7 \\
\hline \multirow{6}{*}{$\begin{array}{l}\stackrel{0}{ \pm} \\
\pm \\
\frac{0}{\omega}\end{array}$} & \multirow{3}{*}{$\begin{array}{c}\text { Speedling } \\
\text { Trays }\end{array}$} & 0 & 344.0 & 2.213 & 47621 & 12.0 & 484.3 & 358.3 & 2.300 & 50229 & 12.0 & 547.7 \\
\hline & & 100 & 323.0 & 2.255 & 49179 & 11.5 & 515.7 & 345.7 & 2.357 & 50683 & 12.6 & 535.3 \\
\hline & & 200 & 348.7 & 2.250 & 45220 & 12.2 & 471.0 & 350.3 & 2.402 & 51728 & 12.6 & 537.0 \\
\hline & \multirow{3}{*}{$\begin{array}{l}\text { Pots } \\
8 \mathrm{~cm}\end{array}$} & 0 & 324.7 & 2.263 & 47345 & 12.6 & 482.7 & 360.3 & 2.383 & 50923 & 13.2 & 537.0 \\
\hline & & 100 & 328.0 & 2.242 & 46437 & 11.8 & 498.7 & 349.7 & 2.327 & 49674 & 13.1 & 529.3 \\
\hline & & 200 & 333.7 & 2.227 & 46070 & 11.6 & 494.3 & 361.7 & 2.407 & 51308 & 12.1 & 549.0 \\
\hline \multirow{6}{*}{ 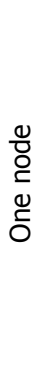 } & \multirow{3}{*}{$\begin{array}{c}\text { Speedling } \\
\text { Trays }\end{array}$} & 0 & 340.0 & 2.205 & 47493 & 12.3 & 508.0 & 361.7 & 2.363 & 51918 & 12.8 & 546.7 \\
\hline & & 100 & 339.0 & 2.303 & 45911 & 12.1 & 508.7 & 355.0 & 2.393 & 51163 & 12.7 & 552.7 \\
\hline & & 200 & 340.3 & 2.287 & 46925 & 11.8 & 526.0 & 343.7 & 2.395 & 52455 & 12.4 & 553.0 \\
\hline & \multirow{3}{*}{$\begin{array}{l}\text { Pots } \\
8 \mathrm{~cm}\end{array}$} & 0 & 336.7 & 2.235 & 46853 & 12.2 & 494.7 & 347.3 & 2.343 & 50000 & 12.5 & 549.0 \\
\hline & & 100 & 338.3 & 2.313 & 44689 & 12.2 & 520.7 & 344.3 & 2.373 & 51463 & 12.8 & 544.7 \\
\hline & & 200 & 339.0 & 2.250 & 46283 & 11.9 & 504.0 & 354.0 & 2.397 & 49726 & 12.8 & 556.0 \\
\hline \multicolumn{3}{|c|}{ L.S.D. 0.05} & NS & NS & NS & NS & NS & NS & NS & NS & NS & NS \\
\hline
\end{tabular}




\section{Flowering}

It is obvious from Table 8 that the watermelon plants obtained from plant cuttings in all cultivars in both seasons showed a significant speedily transformation to flowering (15-20 days) earlier than the watermelon plants produced from seeds (control). This result may be due to the more vigorous vegetative growth than the control during the first 45 days after planting, and also due to significant increment in roots number in all seedlings from all cuttings (Table 1). Early flowering may be due to using the cuttings from adult watermelon plants and the mother plants had exceeded the juvenility stage. Similar results were found by Kurata (1976) and Sakata et al. (2007) they found that grafting watermelon onto bottle gourd caused early formation of female flowers. Flowering date affects fruit harvesting time, which show a direct impact on quality. Sex expression and flowering order are controlled by plant hormones.

These differences in number of days to flowering led to large differences in the time of the harvesting stage between the watermelon plants produced from cuttings compared with the control plants. The harvesting stage started and ended from cuttings 15 days earlier than the control. The early production was due to early flowering in all cutting treatments.

\section{Yield and its components}

Results in Tables 9, 10 and 11 showed that there were significant differences between the plants produced from all cuttings and the control in early yield in all watermelon cultivars in both seasons. Early yield from all cutting treatments showed a significant earliness as compared with the control plants. The highest early yield in Aswan hybrid was recorded in the first season for one node cutting which was treated with 0 ppm IBA followed by planting in speedling trays (5.41ton/feddan), while in the second season one node cuttings treated with 100 IBA and planted in speedling trays gave (5.37 ton/feddan). The differences among all cutting treatments didn't arrive to the significant level. Similarly, all cutting treatments showed a higher early yield from 240.67 to $258.85 \%$ in the first season and 244.44 to $271.21 \%$ in the second season compared with control plants in Aswan cultivar and from 208.46 to $228.57 \%$ in the first season and from 217.64 to $229.94 \%$ ) in the second season in "Buttercup" while this increase in early yield was in the seedless hybrid "SSX7402" from 173.3 to $208.25 \%$ in the first season and 179.24 to $197.64 \%$ in the second season. No significant differences were recorded among all cutting treatments in all cultivars during the two studied seasons. This method of propagation gave positive significant effects on early yield, this may be due to using the mother plants after the flowering stage. Also, it may be due to the strong vegetative growth in the plants which 
produced from all cutting treatments in both seasons. These results are consistent with those reported by El-Eslamboly, (2010). Data in the same tables showed no significant differences in total, marketable yield among all treatments in all cultivars in both seasons.

Data in the same Tables $(9,10$ and 11) illustrated that, no significant differences between all cutting treatments and control concerning, fruit fresh weight, fruit size and rind thickness in both seasons. These characteristics were not affected by this method of propagation in Aswan hybrid.

The red seedless watermelon SSX $7402 F_{1}$ and yellow seedless watermelon Buttercup $F_{1}$ hybrid showed the same trend of results as the seeded watermelon Aswan $F_{1}$ in fruit fresh weight and size and rind thickness. No significant differences were also noticed between all cutting treatments and control plants in fruit weight, size and rind thickness.

Data in Table 12 clearly indicated that there were no significant differences in total chlorophyll content as SPAD values between all treatments during the two seasons as shown in different experiments under this study. SPAD values have been shown to have a strong correlation with leaf chlorophyll content and can be used for rapid diagnosis of leaf $\mathrm{N}$ supply status (Yadava, 1986).Generally no plants showed symptoms of $\mathrm{N}$ deficiency in the present work. SPAD readings may assist in evaluating the status of $\mathrm{N}$ in crops and could potentially define $\mathrm{N}$ fertilizer needs in horticultural crops.

Currently, there are no clear sampling guidelines for using chlorophyll $\mathrm{N}$ levels as an indicator of leaf $\mathrm{N}$ status in a range of crops. The SPAD meter may help growers to optimize $\mathrm{N}$ fertilizer applications to improve tree health and long-term cumulative fruit yield. It is presumed that the increased yield and vegetative growth are due to increases in vigor, chlorophyll content, photosynthesis and peroxidase activity in the plants.

Changes in concentration of leaf pigments (chlorophyll and carotenoids) and its relation are good indicators of perturbations in plants caused by environmental factors. Chlorophyll content was measured as an indicator of the photosynthetic rate (Leidi et al., 1991). The plant analysis diagnostic (SPAD) chlorophyll meter provides a quick and non destructive method for estimating the level of $\mathrm{N}$ in leaves (Leidi et al., 1991). 
Table 8. Effect of using cuttings treated with three concentrations of IBA in two containers compared with true seeds on plant dry weight and number of days to flowering of three watermelon hybrids in 2011 and 2012 seasons.

\begin{tabular}{|c|c|c|c|c|c|c|c|c|c|c|c|c|c|c|}
\hline \multirow{2}{*}{\multicolumn{3}{|c|}{ Characteristics }} & \multicolumn{6}{|c|}{2011} & \multicolumn{6}{|c|}{2012} \\
\hline & & & \multicolumn{3}{|c|}{ Plant dry weight(g) } & \multicolumn{3}{|c|}{ No. days to flowering } & \multicolumn{3}{|c|}{ Plant dry weight(g) } & \multicolumn{3}{|c|}{ No. days to flowering } \\
\hline \multicolumn{2}{|c|}{ Treatments } & $\mathrm{ppm}$ & Buttercup & SSX 7402 & Aswan & Buttercup & $\begin{array}{l}\text { SSX } \\
7402\end{array}$ & Aswan & Buttercup & SSX 7402 & Aswan & Buttercup & $\begin{array}{c}\text { SSX } \\
7402\end{array}$ & Aswan \\
\hline \multicolumn{3}{|c|}{ Control from seeds } & 230.77 & 308.68 & 264.06 & 31.0 & 36.3 & 31.3 & 229.19 & 301.46 & 245.32 & 34.7 & 31.3 & 34.7 \\
\hline \multirow{6}{*}{$\begin{array}{l}\stackrel{ }{D} \\
\stackrel{0}{\circ} \\
\frac{o}{n}\end{array}$} & \multirow{3}{*}{$\begin{array}{c}\text { Speedling } \\
\text { Trays }\end{array}$} & 0 & 226.92 & 301.09 & 242.14 & 16.0 & 17.3 & 17.0 & 229.94 & 302.98 & 244.56 & 14.3 & 13.3 & 15.3 \\
\hline & & 100 & 224.59 & 294.32 & 263.97 & 15.3 & 17.0 & 17.0 & 227.86 & 290.81 & 297.60 & 13.7 & 13.7 & 16.0 \\
\hline & & 200 & 227.14 & 294.09 & 241.18 & 15.0 & 15.7 & 16.7 & 231.17 & 289.3 & 268.80 & 15.3 & 14.3 & 16.3 \\
\hline & \multirow{3}{*}{$\begin{array}{l}\text { Pots } \\
8 \mathrm{~cm}\end{array}$} & 0 & 225.17 & 294.34 & 250.12 & 15.3 & 16.7 & 17.0 & 227.66 & 281.23 & 281.42 & 15.0 & 14.7 & 16.7 \\
\hline & & 100 & 229.37 & 295.53 & 232.57 & 14.7 & 16.0 & 16.3 & 225.52 & 285.47 & 265.14 & 14.3 & 13.7 & 16.0 \\
\hline & & 200 & 224.59 & 287.25 & 253.01 & 15.0 & 15.3 & 17.3 & 231.97 & 291.24 & 270.77 & 16.0 & 15.0 & 16.0 \\
\hline \multirow{5}{*}{$\begin{array}{l}\frac{0}{0} \\
\frac{1}{c} \\
\stackrel{0}{\delta} \\
\text { d. }\end{array}$} & \multirow{2}{*}{$\begin{array}{c}\text { Speedling } \\
\text { Trays }\end{array}$} & 0 & 223.72 & 281.03 & 250.75 & 15.7 & 15.7 & 18.0 & 230.50 & 292.28 & 267.58 & 17.7 & 15.7 & 15.7 \\
\hline & & 200 & 228.35 & 297.35 & 268.36 & 15.3 & 16.7 & 16.7 & 234.74 & 304.12 & 272.80 & 15.7 & 15.0 & 16.3 \\
\hline & \multirow{3}{*}{$\begin{array}{l}\text { Pots } \\
8 \mathrm{~cm}\end{array}$} & 0 & 228.46 & 295.14 & 253.69 & 14.7 & 15.0 & 16.7 & 231.67 & 290.83 & 287.79 & 15.3 & 14.3 & 15.7 \\
\hline & & 100 & 232.21 & 300.49 & 256.75 & 14.7 & 15.3 & 16.7 & 233.04 & 297.14 & 266.33 & 15.3 & 14.3 & 16.3 \\
\hline & & 200 & 229.01 & 294.71 & 276.39 & 15.7 & 16.0 & 17.7 & 232.75 & 291.96 & 274.19 & 14.7 & 14.7 & 16.0 \\
\hline \multicolumn{3}{|c|}{ L.S.D. 0.05} & NS & NS & NS & 3.57 & 4.1 & 3.87 & NS & NS & NS & 4.81 & 3.25 & 3.68 \\
\hline
\end{tabular}


Table 9. Effect of using cuttings treated with three concentrations of IBA in two containers compared with true seeds on yield and fruit characteristics of Aswan hybrid in 2011 and 2012 seasons.

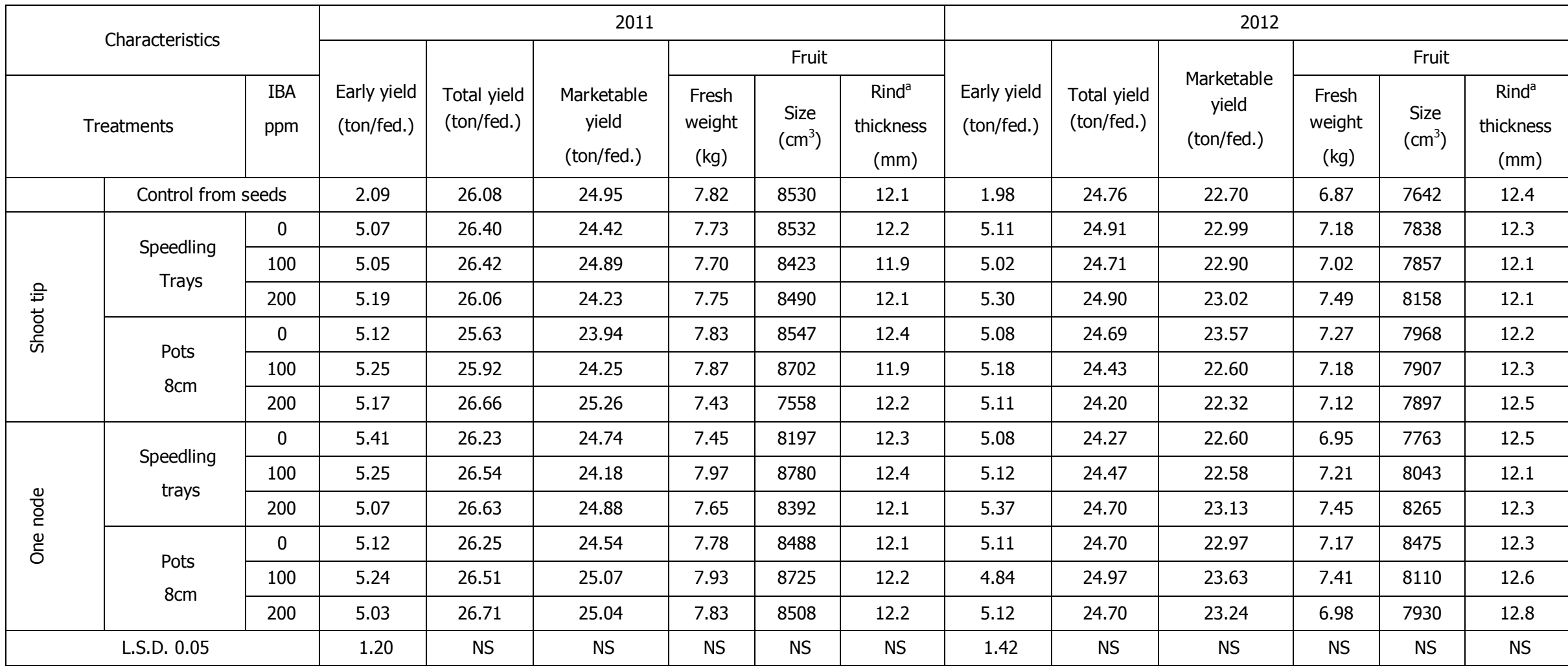

${ }^{\mathrm{a}}$ Rind thickness $=$ rind were measured in four places of the fruit (stem end and thereafter every 90 degrees and an average was taken, average three watermelon fruits). 
Table 10. Effect of using cuttings treated with three concentrations of IBA in two containers compared with true seeds on yield and fruit characteristics of Buttercup hybrid in 2011 and 2012 seasons.

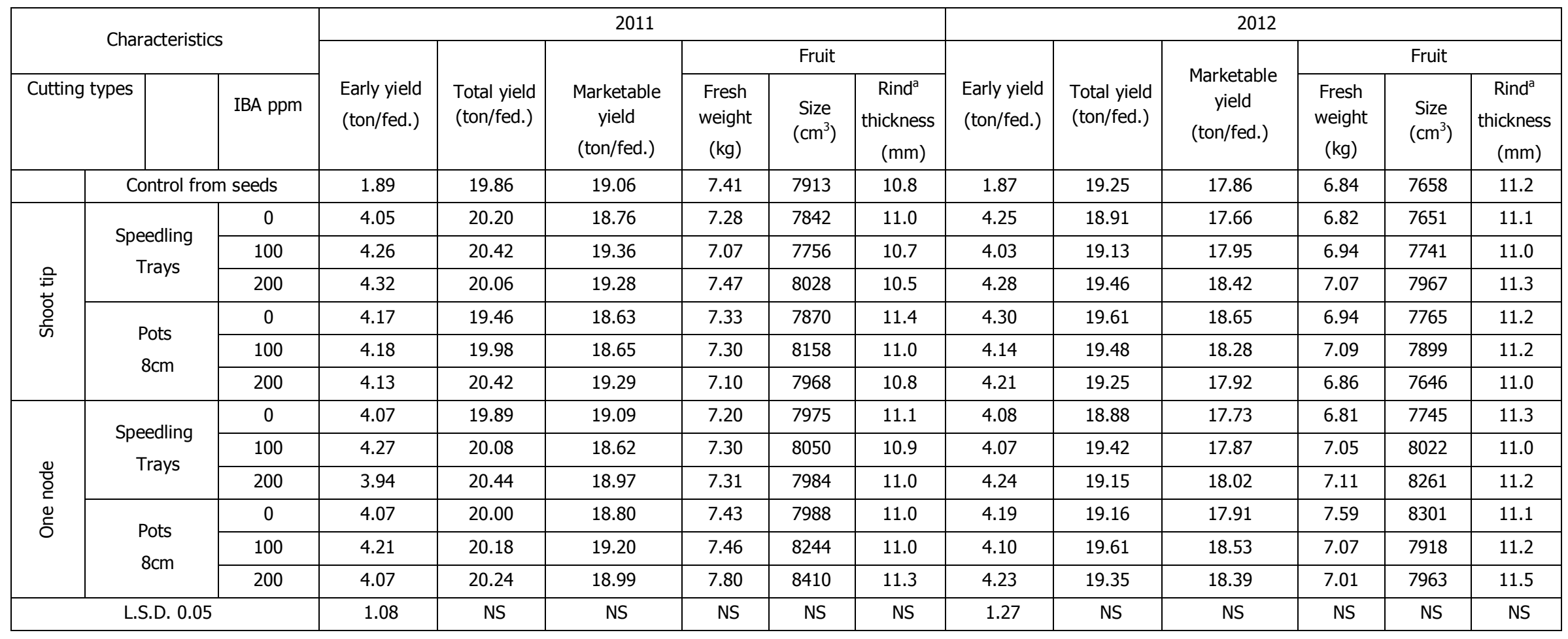

${ }^{\mathrm{a}}$ Rind thickness $=$ rind were measured in four places of the fruit (stem end and thereafter every 90 degrees and an average was taken, average three watermelon fruits). 
Table 11. Effect of using cuttings treated with three concentrations of IBA in two containers compared with true seeds on yield and fruit characteristics of SSX 7402 hybrid in 2011 and 2012 seasons.

\begin{tabular}{|c|c|c|c|c|c|c|c|c|c|c|c|c|c|c|}
\hline \multirow{2}{*}{\multicolumn{3}{|c|}{ Characteristics }} & \multicolumn{6}{|c|}{2011} & \multicolumn{6}{|c|}{2012} \\
\hline & & & \multirow[b]{2}{*}{$\begin{array}{l}\text { Early yield } \\
\text { (ton/fed.) }\end{array}$} & \multirow[b]{2}{*}{$\begin{array}{c}\text { Total yield } \\
\text { (ton/fed.) }\end{array}$} & \multirow{2}{*}{$\begin{array}{l}\text { Marketable } \\
\text { yield } \\
\text { (ton/fed.) }\end{array}$} & \multicolumn{3}{|c|}{ Fruit } & \multirow[b]{2}{*}{$\begin{array}{l}\text { Early yield } \\
\text { (ton/fed.) }\end{array}$} & \multirow[b]{2}{*}{$\begin{array}{l}\text { Total yield } \\
\text { (ton/fed.) }\end{array}$} & \multirow{2}{*}{$\begin{array}{c}\text { Marketable } \\
\text { yield } \\
\text { (ton/fed.) }\end{array}$} & \multicolumn{3}{|c|}{ Fruit } \\
\hline & eatments & $\begin{array}{l}\text { IBA } \\
\text { ppm }\end{array}$ & & & & $\begin{array}{c}\text { Fresh } \\
\text { weight }\end{array}$ & Size $\left(\mathrm{cm}^{3}\right)$ & $\begin{array}{l}\text { Rind }^{\mathrm{a}} \\
\text { thickness }\end{array}$ & & & & $\begin{array}{c}\text { Fresh } \\
\text { weight }\end{array}$ & $\begin{array}{c}\text { Size } \\
\left(\mathrm{cm}^{3}\right)\end{array}$ & $\begin{array}{c}\text { Rind }^{\mathrm{a}} \\
\text { thickness }\end{array}$ \\
\hline & \multicolumn{2}{|c|}{ Control from seeds } & 2.06 & 17.44 & 16.82 & 6.42 & 7059 & 11.6 & 2.12 & 16.82 & 16.45 & 6.29 & 6950 & 12.1 \\
\hline \multirow{6}{*}{$\begin{array}{l}\text { 을 } \\
\text { t。 } \\
\text { 은 }\end{array}$} & \multirow{3}{*}{$\begin{array}{c}\text { Speedling } \\
\text { trays }\end{array}$} & 0 & 3.80 & 17.86 & 16.69 & 6.26 & 7121 & 11.9 & 4.19 & 16.54 & 15.72 & 5.93 & 6298 & 12.0 \\
\hline & & 100 & 3.93 & 18.34 & 17.55 & 5.89 & 6820 & 11.6 & 3.81 & 17.21 & 16.45 & 6.32 & 7137 & 11.9 \\
\hline & & 200 & 4.29 & 17.90 & 18.02 & 6.17 & 6912 & 11.1 & 4.08 & 17.75 & 17.34 & 6.10 & 6892 & 12.6 \\
\hline & \multirow{3}{*}{$\begin{array}{l}\text { Pots } \\
8 \mathrm{~cm}\end{array}$} & 0 & 4.01 & 17.00 & 16.88 & 6.26 & 7166 & 12.4 & 4.05 & 18.27 & 17.30 & 6.07 & 6858 & 12.3 \\
\hline & & 100 & 3.92 & 17.86 & 16.62 & 6.17 & 6986 & 12.3 & 3.89 & 18.26 & 17.46 & 6.45 & 7213 & 12.3 \\
\hline & & 200 & 3.88 & 18.09 & 17.02 & 6.21 & 7055 & 11.5 & 4.12 & 17.98 & 16.95 & 6.08 & 6888 & 11.6 \\
\hline \multirow{6}{*}{$\begin{array}{l}0 \\
\frac{0}{0} \\
\frac{1}{0} \\
0 \\
0\end{array}$} & \multirow{3}{*}{$\begin{array}{c}\text { Speedling } \\
\text { trays }\end{array}$} & 0 & 3.87 & 17.36 & 17.10 & 6.39 & 7089 & 12.0 & 3.85 & 17.11 & 16.26 & 6.14 & 7018 & 12.3 \\
\hline & & 100 & 4.10 & 17.47 & 16.62 & 6.06 & 6890 & 11.6 & 3.80 & 18.10 & 16.58 & 6.35 & 7347 & 12.1 \\
\hline & & 200 & 3.57 & 18.16 & 16.68 & 6.40 & 7133 & 12.1 & 4.21 & 17.25 & 16.36 & 6.21 & 6990 & 12.3 \\
\hline & \multirow{3}{*}{$\begin{array}{l}\text { Pots } \\
8 \mathrm{~cm}\end{array}$} & 0 & 3.79 & 17.57 & 16.66 & 6.50 & 7263 & 12.1 & 4.08 & 17.29 & 16.28 & 6.46 & 7307 & 12.0 \\
\hline & & 100 & 3.99 & 17.72 & 17.02 & 6.41 & 7150 & 12.0 & 4.15 & 18.02 & 16.98 & 6.18 & 7050 & 11.9 \\
\hline & & 200 & 3.89 & 17.66 & 16.59 & 6.20 & 7731 & 12.4 & 4.16 & 16.70 & 17.06 & 6.49 & 7330 & 12.3 \\
\hline \multicolumn{3}{|c|}{ L.S.D. 0.05} & 0.98 & NS & NS & NS & NS & NS & 1.13 & NS & NS & NS & NS & NS \\
\hline
\end{tabular}

${ }^{\text {a }}$ Rind thickness $=$ rind were measured in four places of the fruit (stem end and thereafter every 90 degrees and an average was taken, average three watermelon fruits). 
Table 12. Effect of using cuttings treated with three concentrations of IBA in two containers compared with true seeds on total chlorophyll of three watermelon hybrids in 2011 and 2012 seasons.

\begin{tabular}{|c|c|c|c|c|c|c|c|c|}
\hline \multirow{2}{*}{\multicolumn{3}{|c|}{ Characteristics }} & \multicolumn{3}{|c|}{2011} & \multicolumn{3}{|c|}{2012} \\
\hline & & & \multicolumn{6}{|c|}{ Total chlorophyll (SPAD value) } \\
\hline \multicolumn{2}{|c|}{ Treatments } & IBA ppm & Buttercup & SSX 7402 & Aswan & Buttercup & SSX 7402 & Aswan \\
\hline & \multicolumn{2}{|c|}{ Control from seeds } & 58.56 & 56.90 & 65.21 & 60.53 & 58.20 & 67.01 \\
\hline \multirow{6}{*}{$\begin{array}{l}\stackrel{ }{\equiv} \\
+ \\
\frac{0}{s} \\
\frac{0}{n}\end{array}$} & \multirow{3}{*}{$\begin{array}{c}\text { Speedling } \\
\text { Trays }\end{array}$} & 0 & 63.25 & 57.25 & 67.22 & 59.25 & 57.18 & 66.07 \\
\hline & & 100 & 58.38 & 57.31 & 66.61 & 57.30 & 56.41 & 66.34 \\
\hline & & 200 & 58.89 & 57.27 & 65.52 & 59.87 & 58.37 & 68.04 \\
\hline & & 0 & 57.64 & 56.20 & 66.36 & 57.64 & 56.44 & 66.06 \\
\hline & & 100 & 58.53 & 56.99 & 66.55 & 59.84 & 58.41 & 68.17 \\
\hline & & 200 & 58.61 & 57.11 & 67.20 & 59.01 & 57.57 & 67.16 \\
\hline \multirow{6}{*}{$\begin{array}{l}\frac{0}{0} \\
\frac{1}{c} \\
0 \\
\text { ठ }\end{array}$} & \multirow{3}{*}{$\begin{array}{c}\text { Speedling } \\
\text { Trays }\end{array}$} & 0 & 57.72 & 57.00 & 64.65 & 58.77 & 57.41 & 67.04 \\
\hline & & 100 & 59.71 & 56.75 & 67.39 & 58.86 & 57.70 & 67.59 \\
\hline & & 200 & 59.36 & 57.84 & 67.93 & 58.54 & 56.34 & 64.93 \\
\hline & & 0 & 58.77 & 57.81 & 66.60 & 60.70 & 58.09 & 66.61 \\
\hline & & 100 & 62.20 & 56.94 & 65.21 & 66.86 & 57.13 & 67.33 \\
\hline & & 200 & 58.78 & 56.81 & 65.72 & 59.65 & 57.06 & 65.40 \\
\hline \multicolumn{3}{|c|}{ L.S.D. 0.05} & NS & NS & NS & NS & NS & NS \\
\hline
\end{tabular}




\section{Fruit characteristics}

Data in Tables 13, 14 and 15 illustrated that, this method of propagation had no significant effect on fruit characteristics (concerning TSS., fruit dry matter percentage, total sugar content and panel test) in all watermelon cultivars under this study in both seasons and did not show any harmful effects on fruit quality. The vegetative propagation may give plants genetically identical with the mother plants. In this regard Hartmann et al., (2002) reported that cutting is the most common artificial vegetative propagation method, where pieces of the "parent" plant are removed and placed in a suitable environment. So they can grow into a whole new plant, the "clone", which is genetically identical to the parent. Cutting exploits the ability of plants to grow adventitious roots (i.e. root material that can generate from a location other than the existing or primary root system, as in from a leaf or cut stem) under certain conditions. Vegetative propagation is usually considered a cloning method.

Table 13. Effect of using cuttings treated with three concentrations of IBA in two containers compared with true seeds on fruit characteristics of Aswan hybrid in 2011 and 2012 seasons.

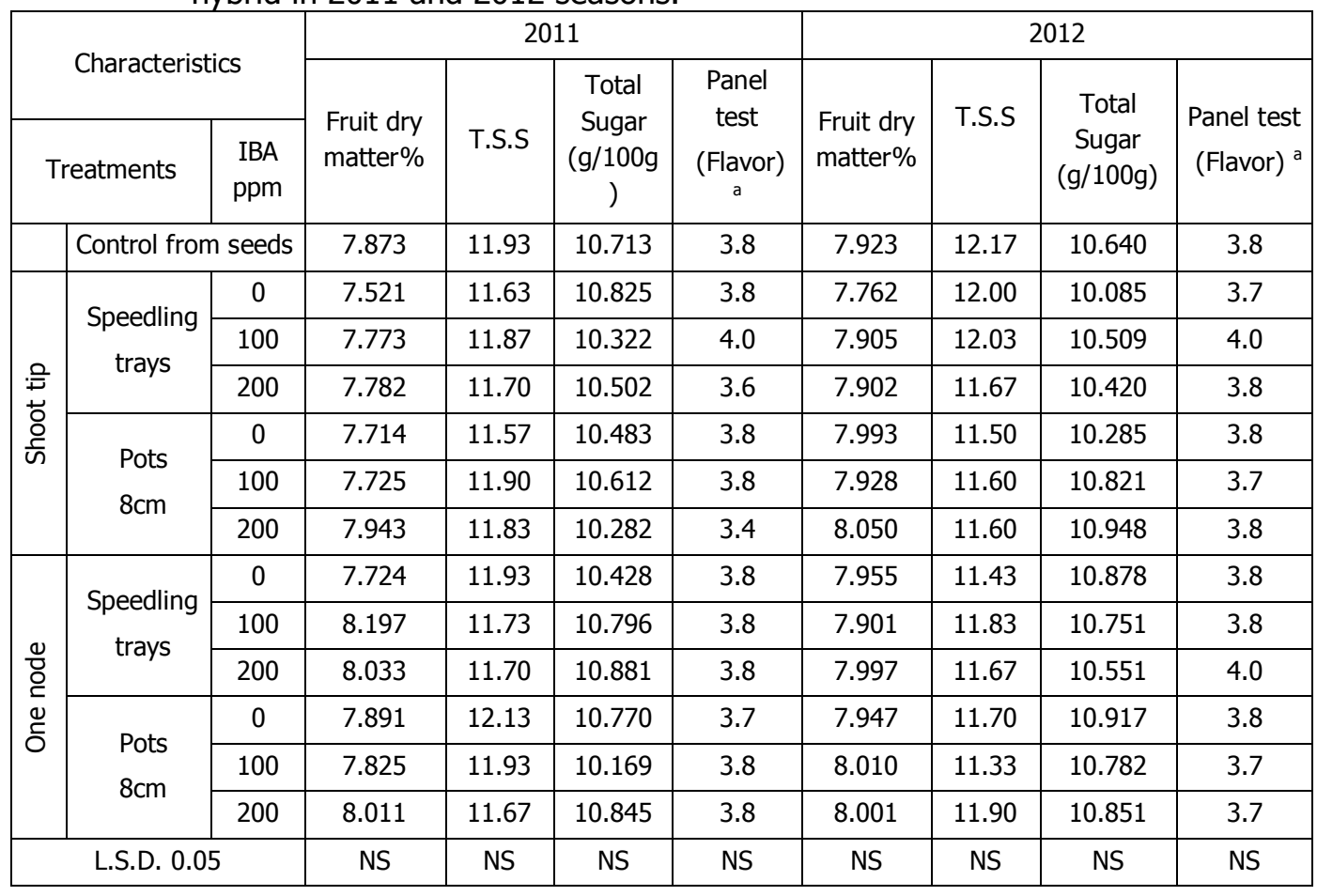

\footnotetext{
a Determined by panel testing (flavor)by 3 persons per replication with 3 replication based on the criteria,
} where $5=$ Excellent, $4=$ Good, $3=$ Medium, 2=Fair and 1= Poor 
Table 14. Effect of using cuttings treated with three concentrations of IBA in two containers compared with true seeds on fruit characteristics of Buttercup hybrid in 2011 and 2012 seasons.

\begin{tabular}{|c|c|c|c|c|c|c|c|c|c|c|}
\hline \multirow{2}{*}{\multicolumn{3}{|c|}{ Characteristics }} & \multirow{3}{*}{$\begin{array}{l}\text { Fruit dry } \\
\text { matter\% }\end{array}$} & \multicolumn{3}{|c|}{2011} & \multicolumn{4}{|c|}{2012} \\
\hline & & & & \multirow{2}{*}{ T.S.S } & \multirow{2}{*}{$\begin{array}{c}\text { Total } \\
\text { sugar } \\
(\mathrm{g} / 100 \mathrm{~g})\end{array}$} & \multirow{2}{*}{$\begin{array}{l}\text { Panel test } \\
\text { (Flavor) }^{\mathrm{a}}\end{array}$} & \multirow{2}{*}{$\begin{array}{l}\text { Fruit dry } \\
\text { matter\% }\end{array}$} & \multirow{2}{*}{ T.S.S } & \multirow{2}{*}{$\begin{array}{c}\text { Total } \\
\text { sugar } \\
(\mathrm{g} / 100 \mathrm{~g})\end{array}$} & \multirow{2}{*}{$\begin{array}{l}\text { Panel test } \\
\text { (Flavor) }^{a}\end{array}$} \\
\hline & atments & $\begin{array}{l}\text { IBA } \\
\text { ppm }\end{array}$ & & & & & & & & \\
\hline & \multicolumn{2}{|c|}{ Control from seeds } & 7.489 & 13.10 & 9.246 & 3.7 & 7.517 & 11.90 & 8.285 & 3.5 \\
\hline \multirow{6}{*}{ 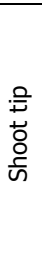 } & \multirow{3}{*}{$\begin{array}{c}\text { Speedling } \\
\text { trays }\end{array}$} & 0 & 7.172 & 12.70 & 8.410 & 3.3 & 7.370 & 12.57 & 9.074 & 3.7 \\
\hline & & 100 & 7.375 & 11.90 & 8.095 & 3.5 & 7.530 & 12.77 & 8.139 & 3.5 \\
\hline & & 200 & 7.252 & 12.67 & 9.183 & 3.3 & 7.392 & 12.47 & 8.211 & 3.5 \\
\hline & \multirow{3}{*}{$\begin{array}{l}\text { Pots } \\
8 \mathrm{~cm}\end{array}$} & 0 & 7.417 & 12.60 & 8.225 & 3.3 & 7.330 & 12.63 & 8.913 & 3.7 \\
\hline & & 100 & 7.375 & 12.53 & 9.184 & 3.5 & 7.382 & 13.07 & 8.313 & 3.5 \\
\hline & & 200 & 7.321 & 12.77 & 8.143 & 3.3 & 7.555 & 12.73 & 8.501 & 3.2 \\
\hline \multirow{6}{*}{$\begin{array}{l}\frac{0}{0} \\
\frac{1}{C} \\
\stackrel{0}{0} \\
0\end{array}$} & \multirow{3}{*}{$\begin{array}{c}\text { Speedling } \\
\text { trays }\end{array}$} & 0 & 7.345 & 12.47 & 9.189 & 3.7 & 7.545 & 12.70 & 8.387 & 3.7 \\
\hline & & 100 & 7.624 & 13.60 & 8.353 & 3.3 & 7.324 & 12.70 & 8.310 & 3.7 \\
\hline & & 200 & 7.539 & 12.80 & 8.373 & 3.7 & 7.496 & 12.67 & 9.253 & 3.3 \\
\hline & \multirow{3}{*}{$\begin{array}{l}\text { Pots } \\
8 \mathrm{~cm}\end{array}$} & 0 & 7.440 & 12.97 & 8.328 & 3.7 & 7.478 & 12.90 & 8.412 & 3.7 \\
\hline & & 100 & 7.482 & 12.63 & 9.062 & 3.3 & 7.350 & 12.97 & 8.881 & 3.7 \\
\hline & & 200 & 7.388 & 12.70 & 8.363 & 3.7 & 7.386 & 12.80 & 8.363 & 3.3 \\
\hline \multicolumn{3}{|c|}{ L.S.D. 0.05} & NS & NS & NS & NS & NS & NS & NS & NS \\
\hline
\end{tabular}

a Determined by panel testing (flavor)by 3 persons per replication with 3 replication based on the criteria, where 5= Excellent, $4=$ Good, 3= Medium, 2=Fair and 1= Poor

Table 15. Effect of using cuttings treated with three concentrations of IBA in two containers compared with true seeds on fruit characteristics of SSX 7402 hybrid in 2011 and 2012 seasons.

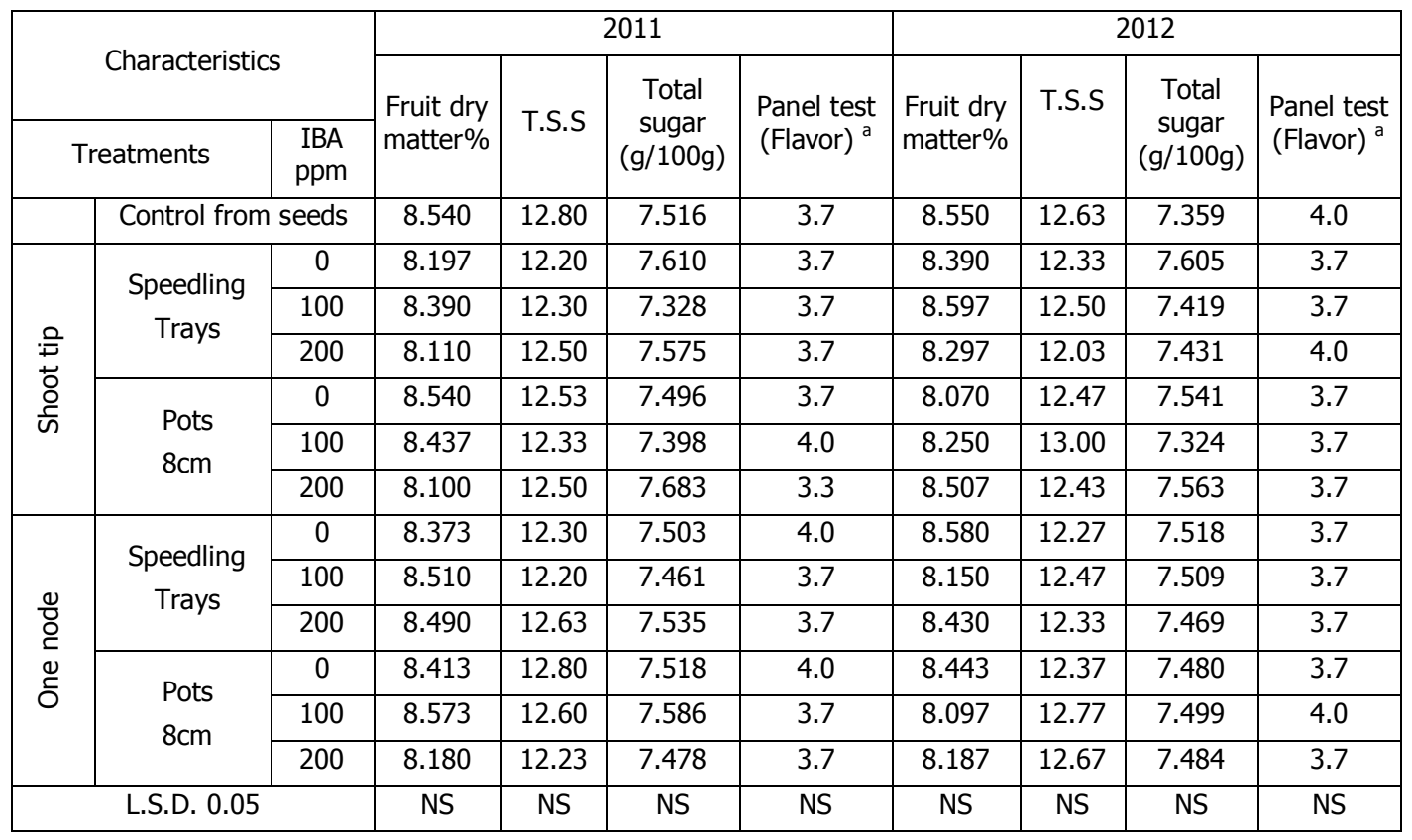

${ }^{a}$ Determined by panel testing (flavor)by 3 persons per replication with 3 replication based on the criteria, where $5=$ Excellent, $4=$ Good, $3=$ Medium, $2=$ Fair and $1=$ Poor 


\section{CONCLUSION}

1. Cutting is a new method for watermelon propagation.

2. The container type had no effect of survival percentage, root number.

3. One node cuttings is best type of cuttings for watermelon propagation.

4. The stem cuttings with one node produced a vigorous plant.

5. The plants from cuttings reached harvest 15 days earlier than the plants from seeds.

6. No significant differences according to total and marketable yield, chlorophyll and fruit characteristics were observed between cutting and seeded watermelon plants.

\section{REFERENCES}

1. Abd El- Hafez, A. A. 1963. Influence of Ploidy of Some Morphological Charactrs in Watermelon. M.Sc. Thesis, Faculty of Agriculture, Cairo University, Egypt, 394 p.

2. Abd El- Hafez, A. A. 1969. Effect of Gene Markers for The Development of Triploid Watermelon Citrullus vulgaris, Schrad. Ph.D. Thesis, Faculty of Agriculture, Cairo University, Egypt, 330 p.

3. Adelberg, J. W., Zhang, X. P. and Rhodes, B. B. 1997. Micropropagation of Citrullus lanatus (Thunb.) Mastsum. and Nakai (Watermelon). pp 60-76. In: Bajaj, Y. P. S. (Ed.) Biotechnology in Agriculture and Forestry, High-Tech Micropropagation V, Vol. 39, Springer-Verlag, Berlin, Heidelerg, ISBN: 3-54061606-3, $401 \mathrm{p}$.

4. Allred Amy, J. and Lucier, G. 1990. The U.S. Watermelon Industry AGES9015. ERS Staff Rep. No. AGES9015, United States Department of Agriculture (USDA). Economic Research Service, Commodity Economic Division. VI, Illinois, 73 p, (Cited by Adelberg et al., 1997).

5. Andrus, C.F., Seshadri, V. S. and Grimbal, P.C. 1971. Production of seedless watermelons. Technical Bulletin, No. 1425. Agricultural Research Service(ARS), United States Department of Agriculture(USDA), Charleston, South Carolina, 12 p.

6. Association of Official Analytical Chemists. 1975. Official Methods of Analysis of the Association of Official Agriculture Chemists. $12^{\text {th }}$ Ed. Published By the A.O.A.C., Washington, D.C., U.S.A, 870p.

7. Davis, A. R., Perkins-Veazie, P., Sakata, Y., López-Galarza, S., Maroto, J. V., Lee, S. G., Huh, Y. C. , Sun, Z., Miguel A., Cohen, S. R. K. R. and Lee J. M. 2008. Cucurbit Grafting. Critical Reviews in Plant Sciences, 27(1): 50 - 74. 
8. El-Abd, M.T.G. 1997. Vegetative propagation of cucumber hybrid 'Cattia'. Egypt journal of horticulture, 24:(1) 59-66

9. El-Eslamboly, A.A.S. 2010. Studies on propagation of watermelon plants. Ph. D Thesis, Department of Vegetable Crops, Faculty of Agriculture, Cairo University Egypt, $151 \mathrm{p}$.

10. Garret, J. J., Rhodes, B. B. and Zhang, X. 1995. Triploid watermelons resist fruit blotch organism. Cucurbits Genetics Cooperation Reports, 18: 56-57.

11. Gomez, K. A. and Gomez, A. A. 1984. Statistical Procedures for Agricultural Research. International Rice Research Institute, $2^{\text {nd }}$ Ed. Joho Wiley and Sons. Inc. New- York, 680 p.

12. Hartmann, H.T., Kester, D. E., Davies, F. T. and Geneve, R. L. 2002. Hartmann and Kester's ,Plant Propagation, Principles and Practices. $7^{\text {th }}$ Edition. New Jersey: Regent-Prentice Hall, 07458. 873 p.

13. Kihara, H. 1951. Triploid watermelon. Proceeding of the American Society for Horticultural Science, 58:217-230.

14. Kurata, H. 1976. Studies on the sex expression of flowers induced by day-length and temperature in pumpkin and watermelon. Memoirs Faculty of Agriculture, Kagawa University, 29: 1-49. (Cited by Davis et al., 2008).

15. Robbins J.A., Kays S.J. and Dirr MA. 1983. Enhanced rooting of wounded mung bean cuttings by wounding and ethephon. Journal of the American Society for Horticultural Science, 108: 325-329.

16. Leidi E.O., Silberbush, M. and Lips, S. H. 1991. Wheat growth as affected by nitrogen type, $\mathrm{pH}$ and salinity. II. Photosynthesis and transpiration. Journal of Plant Nutrition, 14: 247-256.

17. Malik, C. P. and Singh, M. B. 1980. Plant Enzymology and Histoenzymology. A Text Manual. Kalyani Publishers, New Delhi, 262 p.

18. Mason, J.(2004). Nursery management. $2^{\text {th }}$ Edition. ISBN 0643092137 (NetLibrary eBook). Landlinks Press, Collingwood VIC 3066 Australia, 320 p.

19. Sakata, Y., Takayoshi, O. and Mitsuhiro, S. 2007. The history and present state of the grafting of cucurbitaceous vegetables in Japan. Acta Horticulturae, 731: 159170.

20. Yadava, I.L. 1986. A rapid and nondestructive method to determine chlorophyll in intact leaves. HortScience, 21: 1449. 


\section{تأثثر إكثار البطيخ بالعقل علي النمو الخضري والمحصول وجودة الثمار}

\section{احمد عبدالهادي سيد عبد الوهاب الاسلامبولي}

قسم بحوث انتاج الخضر تحت ظروف جوية معدلة، معهد بحوث البساتين، مركز البحوث الزراعية

البطيخ من محاصيل الخضر الهامة في مصر • يفضل عديد من المستهلكين البطـيخ عــديم

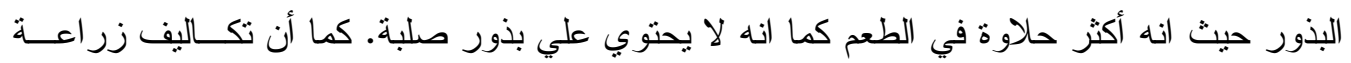

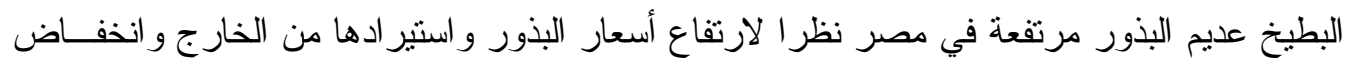

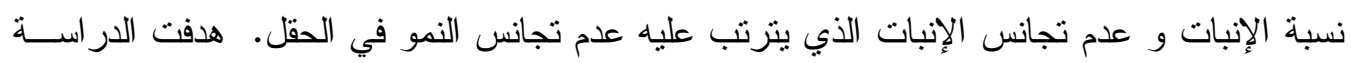

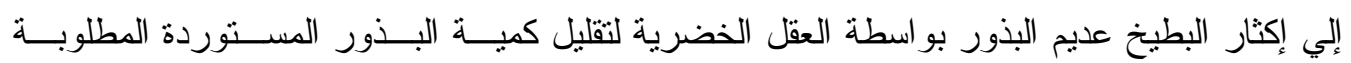

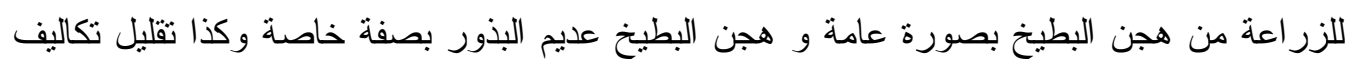

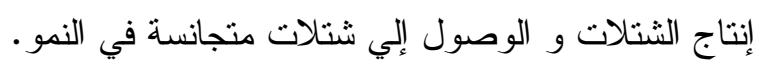

العقل الخضرية طريقة لإكثار النباتات لا جنسيا تستخدم علي نطاق واســع فــي البســاتين

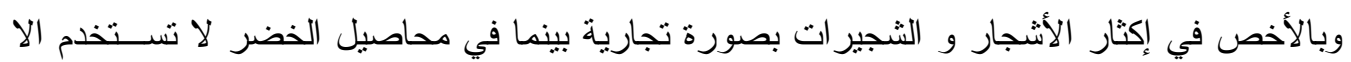

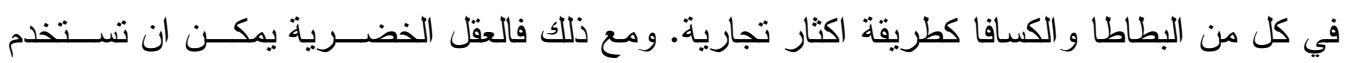

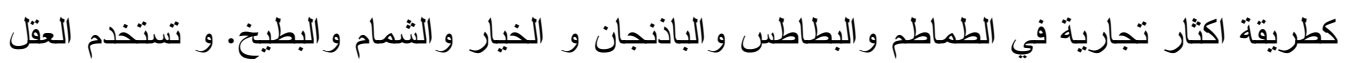
النباتية في هذه الدر اسة كطريقة للاكثار في نباتات البطيخ.

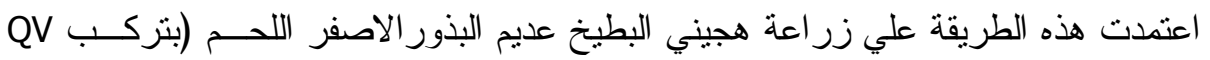

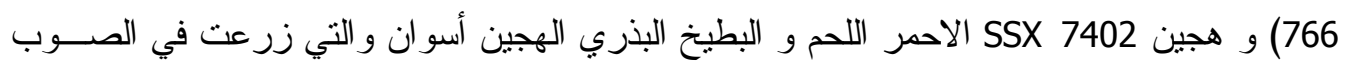

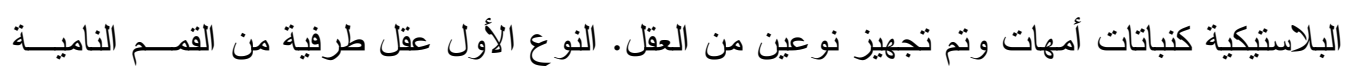

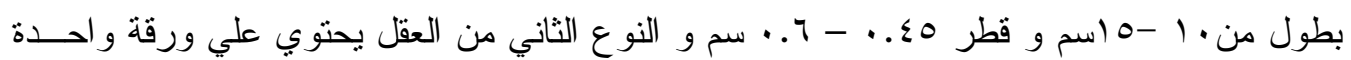
وبر عم إبطي واحد وعقدة واحدة من الهجن الثناث.

تمت الدراسة في ثلاثة تجارب منفصلة كل هجين من هجن البطيخ السالف ذكرها كل فئسي

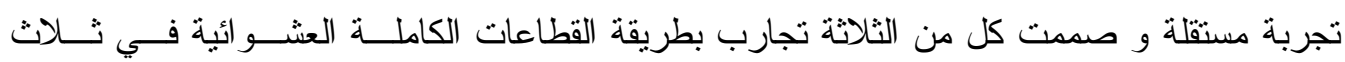

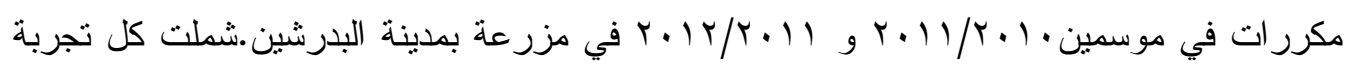

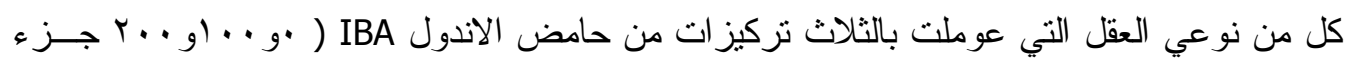

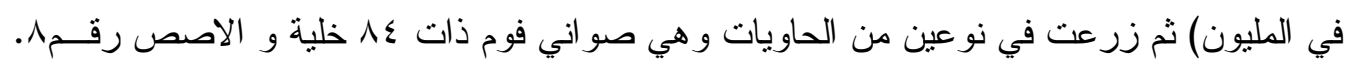

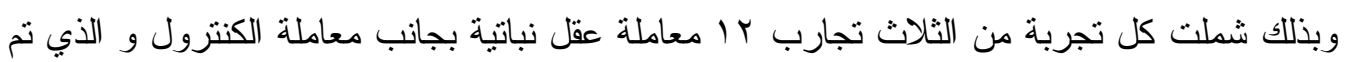
زر اعتة بالبذور .

وقد اوضحت الدراسة ان كل العقل النباتية المشتملة علي قمة نامية (العقل الطرفية) و التي عوملت باي من الثركيز ات الثلاث من IBA في كل من نوعي الحاويات كانت اقل في نسبة البقـاء

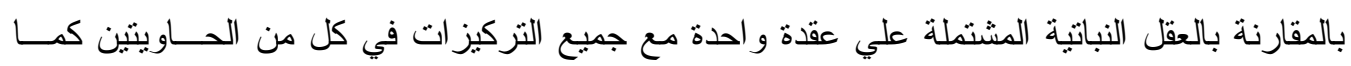


اوضحت زيادة كبيرة في عدد الجذور المتكونة علي العقل المحتوية علي عقدة واحدة بالمقارنة بعـدد الجذور المتكونة علي العقل الطرفية و الكنترول.

جميع معاملات العقل ذات العقدة الواحدة اعطت معدلات بقاء اعلي عن نسب الانبات فــي

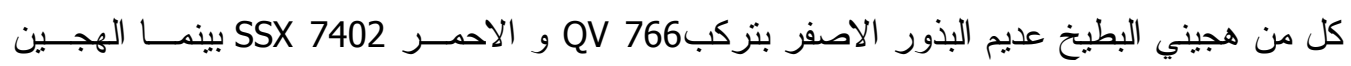

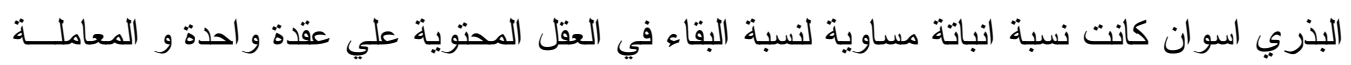

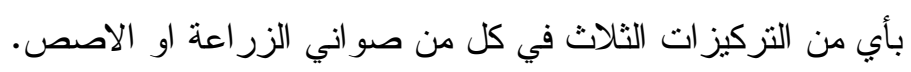

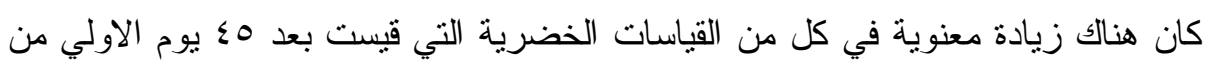

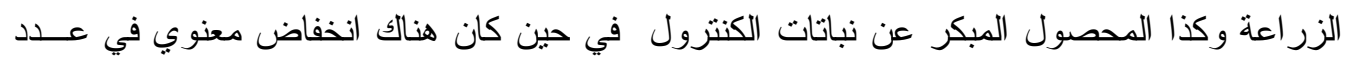

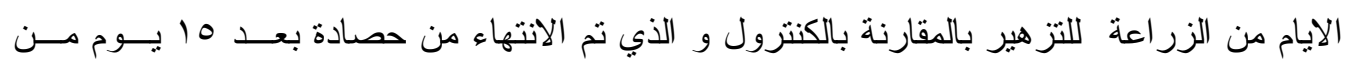

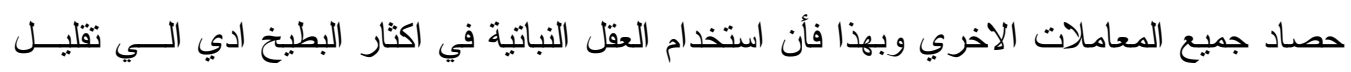
الفترة اللازمة من الزر اعة للحصاد.

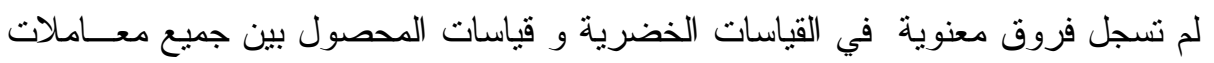

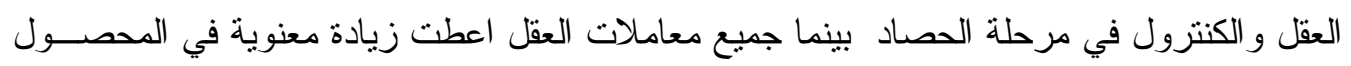

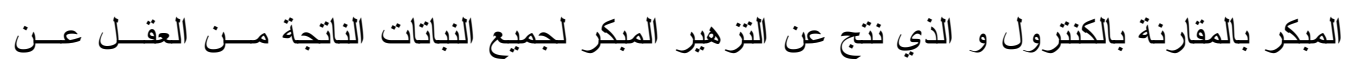
بناتات الكنتزول. الكلمات الدالة : البطيخ عديم البذور - الثلاثي - العقل - الاكثار 